\title{
Intratumoural administration and tumour tissue targeting of cancer immunotherapies
}

\author{
Ignacio Melero $\mathbb{1}^{1,2,3,4,5 凶}$, Eduardo Castanon ${ }^{1,2}$, Maite Alvarez $\mathbb{1}^{3,4,5}$, Stephane Champiat ${ }^{6,7,8}$ \\ and Aurelien Marabelle $\mathbb{1 0}^{6,7,8 凶}$
}

Abstract | Immune-checkpoint inhibitors and chimeric antigen receptor (CAR) T cells are revolutionizing oncology and haematology practice. With these and other immunotherapies, however, systemic biodistribution raises safety issues, potentially requiring the use of suboptimal doses or even precluding their clinical development. Delivering or attracting immune cells or immunomodulatory factors directly to the tumour and/or draining lymph nodes might overcome these problems. Hence, intratumoural delivery and tumour tissue-targeted compounds are attractive options to increase the in situ bioavailability and, thus, the efficacy of immunotherapies. In mouse models, intratumoural administration of immunostimulatory monoclonal antibodies, pattern recognition receptor agonists, genetically engineered viruses, bacteria, cytokines or immune cells can exert powerful effects not only against the injected tumours but also often against uninjected lesions (abscopal or anenestic effects). Alternatively, or additionally, biotechnology strategies are being used to achieve higher functional concentrations of immune mediators in tumour tissues, either by targeting locally overexpressed moieties or engineering 'unmaskable' agents to be activated by elements enriched within tumour tissues. Clinical trials evaluating these strategies are ongoing, but their development faces issues relating to the administration methodology, pharmacokinetic parameters, pharmacodynamic end points, and immunobiological and clinical response assessments. Herein, we discuss these approaches in the context of their historical development and describe the current landscape of intratumoural or tumour tissue-targeted immunotherapies.

Immunity and inflammation constitute physiological responses that have evolved to act more effectively in a tissue-localized fashion; systemic inflammation is usually detrimental and can often be life-threatening. Therefore, immune responses are usually directed to exert maximal effects at the point of entry of infection via the production of particular immunoglobulin isotypes, engagement of tissue-resident $\mathrm{T}$ cells, and selective migration of $\mathrm{T}$ cells and antigen-presenting cells to the affected tissue and/or the draining lymph nodes.

Cancer immunotherapy is advancing at a rapid pace, building on the success of monoclonal antibodies (mAbs) targeting the inhibitory immune-checkpoint proteins PD-1, PD-L1 and CTLA4 (REF.') and adoptive $\mathrm{T}$ cell therapies, particularly chimeric antigen receptor (CAR) $\mathrm{T}$ cells ${ }^{2,3}$. All of these treatments, which have attained regulatory approval for various indications, are infused intravenously and rely on systemic biodistribution for delivery of the active agent to malignant tissue.
Systemic parenteral treatment administration has unequivocal advantages, including predictable serum pharmacokinetics and the simplicity and widespread availability of the required infrastructure, and is therefore a conventional approach used in drug development. However, this mode of delivery also has several limitations and disadvantages (FIG. 1). For example, penetration of macromolecules and cells from the circulation into solid tumours is often limited, which can result in target under-occupancy ${ }^{4,5}$. Moreover, systemic agents often result in systemic toxicities, which might prevent the use of optimal doses. Indeed, intravenous immunotherapies often cause systemic inflammation and autoimmune or autoimmune-like reactions ${ }^{6}$ that probably reflect on-target, off-tumour effects in various non-malignant tissues; this problem has precluded the development and maximally efficacious dosing of immunomodulatory agents, such as IL-12 (REF.') and the anti-CTLA4 $\mathrm{mAb}$ ipilimumab, respectively ${ }^{8}$. Furthermore, systemic 


\section{Key points}

- Repeated intratumoural injections with agents designed to enhance antitumour immune responses constitutes a feasible strategy to reduce the risk of systemic toxicities and achieve higher local bioactive drug concentrations.

- Spearheaded by the oncolytic virus talimogene laheparepvec, the first intratumoural immunotherapy approved by the FDA and EMA, and supported by a strong preclinical rationale, many intratumoural immunotherapies are now being developed in clinical trials.

- These immunotherapies include microorganisms (viruses or bacteria) and synthetic compounds mimicking infectious agents (such as pattern recognition receptor agonists), as well as immunomodulatory monoclonal antibodies, cytokines and chimeric proteins.

- Higher locoregional concentrations of immunotherapy agents can also be achieved through molecular engineering, for example, to target them towards moieties that are enriched in the tumour microenvironment.

- Increased specificity in tumour targeting can also be attained through the development of prodrug forms of immunotherapies that become functional only after entering tumour tissue (pro-immunodrugs).

- Procedural, pharmaceutical, regulatory and analytical challenges require multidisciplinary expert consensus and systematic research to maximize the potential of these modes of administration.

homeostasis can quickly counteract pro-inflammatory and/or immunostimulatory effects of immunotherapies, thus limiting their antitumour activity (for example, tachyphylaxis mediated by target internalization has been observed with $\mathrm{mAb}$ agonists of $\mathrm{OX} 40)^{9}$. These limitations can potentially be overcome by selectively enhancing the bioavailability of immunotherapy agents in the tumour microenvironment (TME), which can be achieved through two strategies: (1) repeated or continuous direct intratumoural delivery, and (2) systemic administration of drugs or prodrugs that selectively accumulate or function in tumour tissues and perhaps also the draining lymphatic tissues (FIG. 1).

Intratumoural administration using image-guided injection is feasible in most organs ${ }^{10}$. Agents administered in this manner will first diffuse throughout the injected area, thereby achieving a very high initial tissue concentration locally, before dissipating into the systemic circulation over time. This gradual absorption into the blood can have pharmacokinetic advantages that permit higher doses with better tolerability, as has been reported with subcutaneous versus intravenous administration of the IL-15 superagonist ALT-803 $\left(\right.$ REF $\left.^{11}\right)$ and the agonistic anti-CD40 mAb selicrelumab (NCT02304393) ${ }^{12}$. Importantly, intratumoural delivery also offers the advantage of immediate access of the agent to tumour-draining lymph nodes, which are

\footnotetext{
Author addresses

${ }^{1}$ Department of Immunology, Clínica Universidad de Navarra, Pamplona, Spain.

2Department of Oncology, Clínica Universidad de Navarra, Pamplona, Spain.

${ }^{3}$ Program for Immunology and Immunotherapy, Center for Applied Medical Research (CIMA), Universidad de Navarra, Pamplona, Spain.

${ }^{4}$ Navarra Institute for Health Research (IdiSNA), Pamplona, Spain.

${ }^{5}$ Centro de Investigación Biomédica en Red de Cáncer (CIBERONC), Madrid, Spain.

${ }^{6}$ Département d'Innovation Thérapeutique et d'Essais Précoces (DITEP), Université Paris

Saclay, Gustave Roussy, Villejuif, France.

${ }^{7}$ INSERM U1015, Gustave Roussy, Villejuif, France.

${ }^{8}$ Biotherapies for In Situ Antitumor Immunization (BIOTHERIS), Centre d'Investigation

Clinique INSERM CICBT1428, Villejuif, France.
}

considered a key hub for the initiation and maintenance of antitumour immune responses ${ }^{13}$. Similarly, local delivery might provide direct access to organized tertiary lymphoid structures within the tumour tissue ${ }^{14,15}$.

Immunotherapy is typically based on the assumption that most tumours harbour non-self antigens (neoantigens) that can be recognized by the immune system $^{16}$. Most tumours are, however, profoundly nonimmunogenic or foster immunological ignorance or even tolerance ${ }^{17}$. Intratumoural injection of proinflammatory agents might provide local adjuvant activity to turn the lesion (or lesions) into an 'in situ cancer vaccine ${ }^{10}$, potentially inducing immunity against tumour antigens shared by most or all metastatic and micrometastatic foci in the patient.

With regard to selectively focusing the biodistribution of systemic agents to tumours, various targeting strategies could be used. For example, active immunotherapy agents can be chimerized to molecules that bind with high affinity to moieties restricted to or enriched in tumour cells, stromal cells or the extracellular matrix of malignant tissues. Combining this approach with intratumoural delivery might further enhance the local bioactive concentrations, given that the targeted agent will be retained within the tumour tissue at least up to the level of target saturation.

Innovative pharmaceutical and biomedical technologies can facilitate the selective delivery of drugs to tumours. Lipid-based microvesicles or nanovesicles are perhaps the most promising approach to enhancing intratumoural bioavailability through slow release of immunotherapies. Alternatively, or additionally, inactive forms of various compounds could be generated as prodrugs that would become selectively activated over time after reaching malignant tissues through systemic biodistribution. This principle of selective prodrug activation relies on characteristic pathobiological or physicochemical properties of the TME, such as a low $\mathrm{pH}$, high concentrations of ATP or overexpression of proteases. Notably, once activated, the compound might drain to lymph nodes. In a variation of this principle, viruses could be engineered to selectively replicate in and destroy tumour tissues or to selectively express transcriptionally targeted transgenes in cancer cells ${ }^{18}$. For example, viruses could be armed with a variety of immune transgenes, such as granulocyte-macrophage colony-stimulating factor (GM-CSF), FLT3 ligand, anti-CTLA4 mAbs or co-stimulatory ligands ${ }^{18}$.

Despite presenting new challenges, intratumoural administration and tumour tissue-targeted delivery have substantial potential to improve immunotherapy (FIG. 1). With numerous active clinical trials ongoing (FIG. 2), we review the current landscape of intratumoural and tumour tissue-targeted immunotherapies.

\section{Historical perspective}

Empirical observations made by William Coley in the late 19th century correlated erysipelas infection with spontaneous regression of sarcoma ${ }^{19}$. On the basis of these observations, patients with a range of malignancies, including soft-tissue sarcoma, were locally inoculated with either live Streptococcus pyogenes or 'Coley 
toxins', consisting of a mixture of killed S. pyogenes and Serratia marcescens, and several had durable tumour regression. Intratumoural administration of Coley toxins was generally safe and had at least some clinical activity ${ }^{20}$. No scientific evidence was presented regarding a

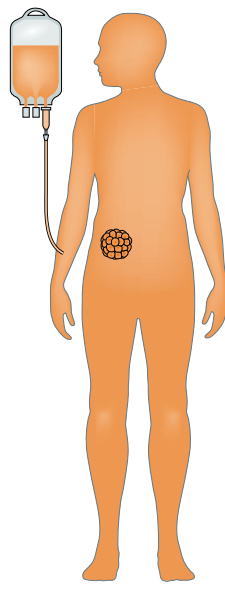
Systemic

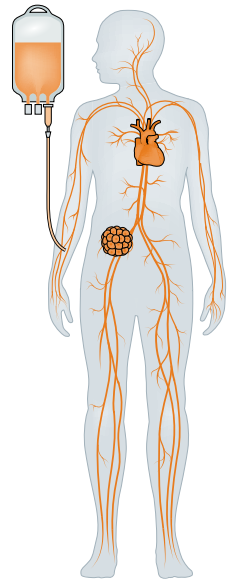

Tumour-targeted

Practical

On-target

off-tumour toxicity

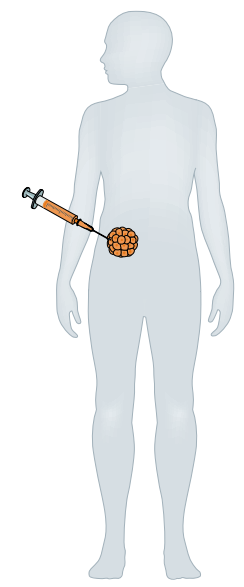

Intratumoural

- High therapeutic index - Low toxicity

Technical
Disadvantages

- Low therapeutic

- High toxicity

b SWOT analysis of intratumoural immunotherapy

Internal
Strengths
- Increased intratumoural bioavailability
(therapeutic index)
- Lower systemic exposure, reducing the
risk of irAEs
- Enables the use of multiple synergistic
combinations

combinations

\section{Weaknesses}

- Requirements relating to logistics, staffing, beds and other resources need to be addressed to implement and increase the treatment capacity and quality in clinical trials of intratumoural immunotherapy

- Skilled radiologists or interventional radiologists are required for the treatment of deep-seated tumours

\section{External}

\section{Opportunities}

- Current boom in local immunotherapy studies, including first-in-human, phase I-III, industry-sponsored and academic trials

- Amenable to longitudinal and multisite studies

- Early pharmacodynamics readouts are possible

Fig. 1 | Strengths, weaknesses, opportunities and threats of intratumoural delivery of immunotherapies. a | Comparison of immunotherapy delivery strategies graphically depicting the typical biodistribution of intravenously administered systemic and tumour tissue-targeted immunotherapies and intratumourally administered immunotherapies. Intravenous delivery has certain practical advantages but also carries a higher risk of adverse events, particularly on-target, off-tumour toxicities related to systemic exposure to the active compound. On the contrary, intratumoural delivery presents technical and logistical challenges but can increase the therapeutic index of immunotherapies within the treated lesions, typically with a low risk of on-target, off-tumour toxicities. b | Summary of the internal strengths and weaknesses as well as external opportunities and threats (SWOT analysis) of intratumoural immunotherapy, all of which need to be balanced against the current clinical drug development landscape of cancer immunotherapy, which encompasses a multitude of novel agents. irAEs, immune-related adverse events; itRECIST, Response Criteria for Intratumoral Immunotherapy in Solid Tumors. the mechanism of action, albeit a key role of the immune system has been postulated ${ }^{21}$. These experiences are reminiscent of the legendary miraculous healing of a tumour in the leg of Saint Pelegrine Laziosi following an infection of the neoplasm ${ }^{22}$.

Inspired by the ideas of Coley, intravesical instillation of bacillus Calmette-Guérin (BCG) was explored in the treatment of superficial bladder cancers ${ }^{23}$ and was found to prolong progression-free survival compared with intracystic chemotherapy ${ }^{24}$. Intravesical BCG remains the standard-of-care treatment for non-muscle-invasive bladder cancer (NMIBC), although patients are currently facing a BCG manufacturing shortage ${ }^{25}$. Mechanistic studies have revealed the importance of an antitumour immune response mediated by IFN $\gamma$-producing $\mathrm{T}$ cells and natural killer (NK) cells as well as activated macrophages for the efficacy of $\mathrm{BCG}^{26}$. In patients with advanced-stage melanoma, intratumoural injection of BCG into accessible lesions elicits tumour regressions, which importantly involved not only the injected lesions but sometimes also uninjected ones ${ }^{27}$. Immune cell infiltration into and surrounding the injected lesions was considered to be an explanation for the apparent clinical activity of this approach ${ }^{27,28}$. Discouraging results in the adjuvant setting halted the development of BCG for the treatment of melanoma, although post-surgical intradermal, rather than intratumoural, administration was used in this setting ${ }^{29,30}$.

Conceptually, intratumoural immunotherapies can act at each step of the cancer immunity cycle conceptualized by Chen and Mellman ${ }^{31}$, but only locally; therefore, a resultant systemic antitumour immune response is required for anenestic or abscopal effects against distant macrometastatic or micrometastatic lesions (FIG. 3).

\section{Intratumoural immunotherapies \\ Pattern recognition receptor agonists}

Infection can be detected by immune cells and non-immune cells via receptors recognizing biomolecules, termed pathogen-associated molecular patterns (PAMPs), that are present in microbes but not animals ${ }^{32,33}$. These pattern recognition receptors (PRRs) are crucial for initiating and sustaining innate and adaptive immune responses. The main innate PRRs for PAMPs include the Toll-like receptor family (TLR), the RIG-I-like receptor (RGR) family of cytoplasmic RNA helicases and the cyclic GMP-AMP synthase (cGAS)-stimulator of interferon genes (STING) system. Evolution seems to have shaped the defences against microbes to detect localized infection and/or tissue damage, thereby preventing systemic infection ${ }^{32,33}$. Intratumoural immunotherapy with PRR agonists aims to induce or enhance local inflammation and immunity in the tumour by mimicking the type of intracellular microbes (virus or bacteria) that evoke cytotoxic $\mathrm{CD}^{+} \mathrm{T}$ cell responses and stimulate $\mathrm{CD} 4^{+}$ T cells to produce IFN $\gamma^{33}$.

TLR9 agonists. TLR9 is present inside endosomes of myeloid cells, B cells and plasmacytoid dendritic cells (pDC), among other immune cells, and recognizes contiguous $\mathrm{CpG}$ dinucleotide sequences in bacterial or viral double-stranded DNA (dsDNA) that, contrary to such 
a

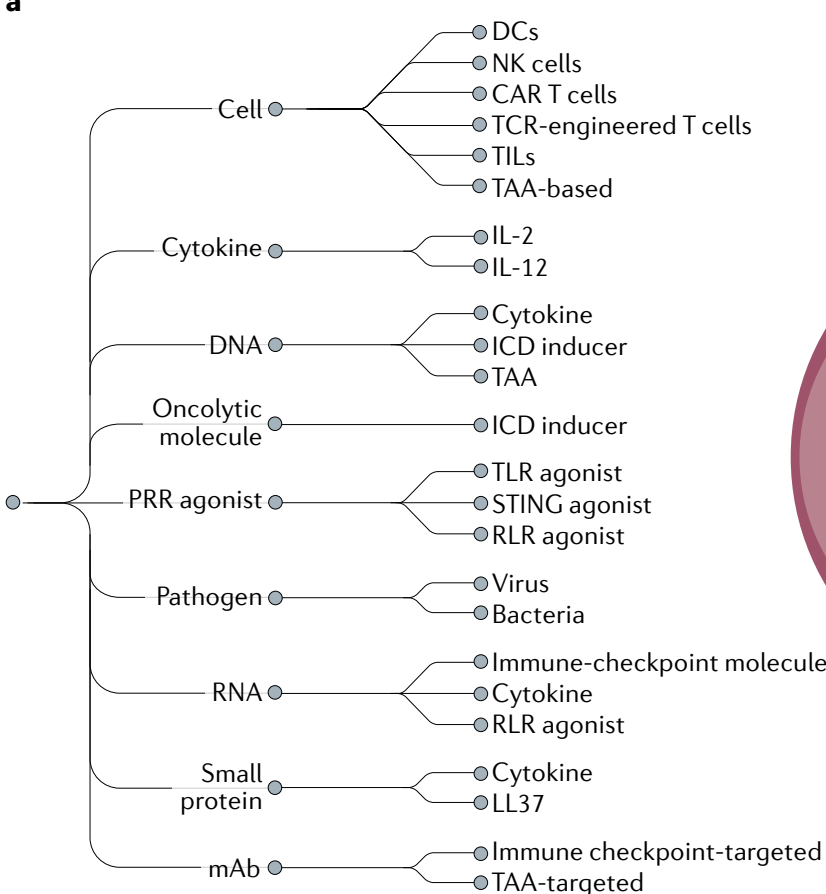

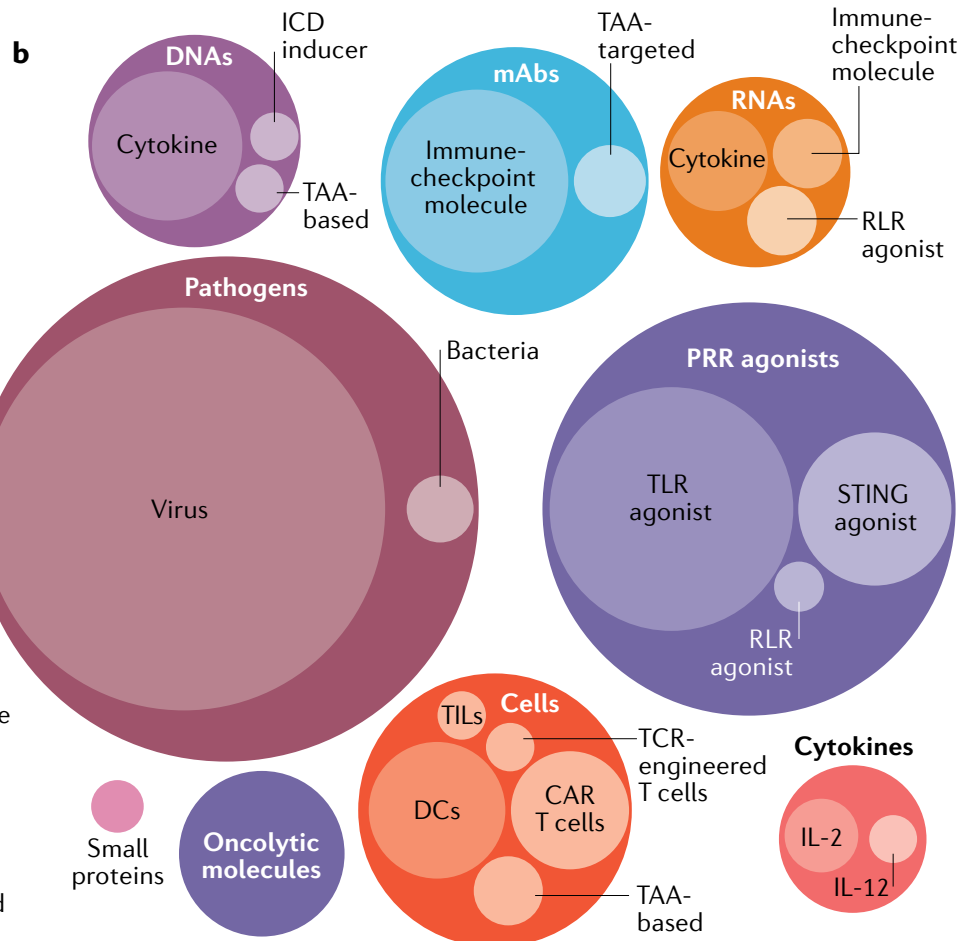

Fig. 2 | Current landscape of active clinical trials of intratumoural immunotherapies. a | Classification of the different types of immunotherapy agents that are currently being investigated in clinical trials involving intratumoural administration as of 1 December 2020 (data obtained from the ClinicalTrials.gov database using the search term "intratumoral OR intralesional AND cancer AND immunotherapy”). b | Visualization of the number of clinical trials for each type of agent outlined in the classification by circle packing, whereby the circle diameter indicates the relative proportion of ongoing or completed clinical trials identified. The trials included in this figure are listed in Supplementary Tables 1-7. CAR, chimeric antigen receptor; DCs, dendritic cells; ICD, immunogenic cell death; LL37, 37-residue cathelicidin antimicrobial peptide; mAb, monoclonal antibody; NK, natural killer; PRR, pattern recognition receptor; RLR, RIG-I-like receptor; STING, stimulator of interferon genes; TAA, tumour-associated antigen; TCR, T cell receptor; TILs, tumour-infiltrating lymphocytes; TLR, Toll-like receptor.

sequences in mammalian DNA, are non-methylated ${ }^{34}$. TLR9 agonists comprising short, contiguous CpG oligonucleotides have a spectrum of pro-inflammatory activities $^{35}$ and can be classified into three categories depending on their structure and the most abundant cytokines they elicit: type A, which predominantly induce the type I interferons IFN $\alpha / \beta$; type B that induce TNF, IL-12, IFN $\gamma$ and IL-6; and type C that induce both sets of cytokines ${ }^{36}$. Type $\mathrm{C}$ oligonucleotides are considered most suitable for antitumour immunity.

Pioneering studies of TLR agonists for cancer immunotherapy utilized a series of CpG oligonucleotides formulated for subcutaneous injection ${ }^{37}$. Following promising results from a randomized phase II trial of the subcutaneous TLR9 type B agonist oligodeoxynucleotide PF-3512676 in combination with chemotherapy for first-line treatment of advanced-stage non-small-cell lung carcinoma (NSCLC) $)^{38}$, further development of this treatment approach was halted owing to negative results of phase III trials ${ }^{39,40}$. However, TLR9 agonists eliciting IFN $\gamma$ and IFN $\alpha / \beta$ responses have been revisited for intratumoural administration.

Intratumoural use of CpG oligonucleotide TLR9 agonists was pioneered by the group of R. Levy in patients with indolent lymphomas or mycosis fungoides ${ }^{41,42}$. This approach was first applied in patients with follicular lymphoma, following low-dose local irradiation of a single pathological lymph node lesion that was to be injected. In one series of 15 patients treated with CpG7909, four $(27 \%)$ had an objective response ${ }^{39}$. More recently, 29 patients were treated with SD-101, of whom six (21\%) had an objective response (as per the revised response criteria for malignant lymphoma); regression of the injected tumour occurred in almost all patients, with regression of uninjected lesions also noted in 24 patients $(83 \%)^{43}$. No severe toxicities were reported in either study.

In patients with advanced-stage melanoma, intratumoural injections of SD-101 have been safely combined with systemic pembrolizumab, and objective responses were seen in $2(15 \%)$ of 13 patients previously refractory to anti-PD-1 mAb monotherapy and 7 (78\%) of 9 patients naive to immunotherapy ${ }^{44}$. This series of patients is being extended, and similar strategies are being tested in patients with melanoma or various other malignancies (Supplementary Table 1).

In both the lymphoma and melanoma studies ${ }^{43,44}$, evidence of increased tumour infiltration by $\mathrm{T}$ cells was observed following therapy. In the case of lymphoma, malignant cells express TLR9 and are therefore likely to respond to the intratumoural CpG oligonucleotide, perhaps with enhanced antigen-presentation ability and thus increased immunogenicity ${ }^{45}$. 


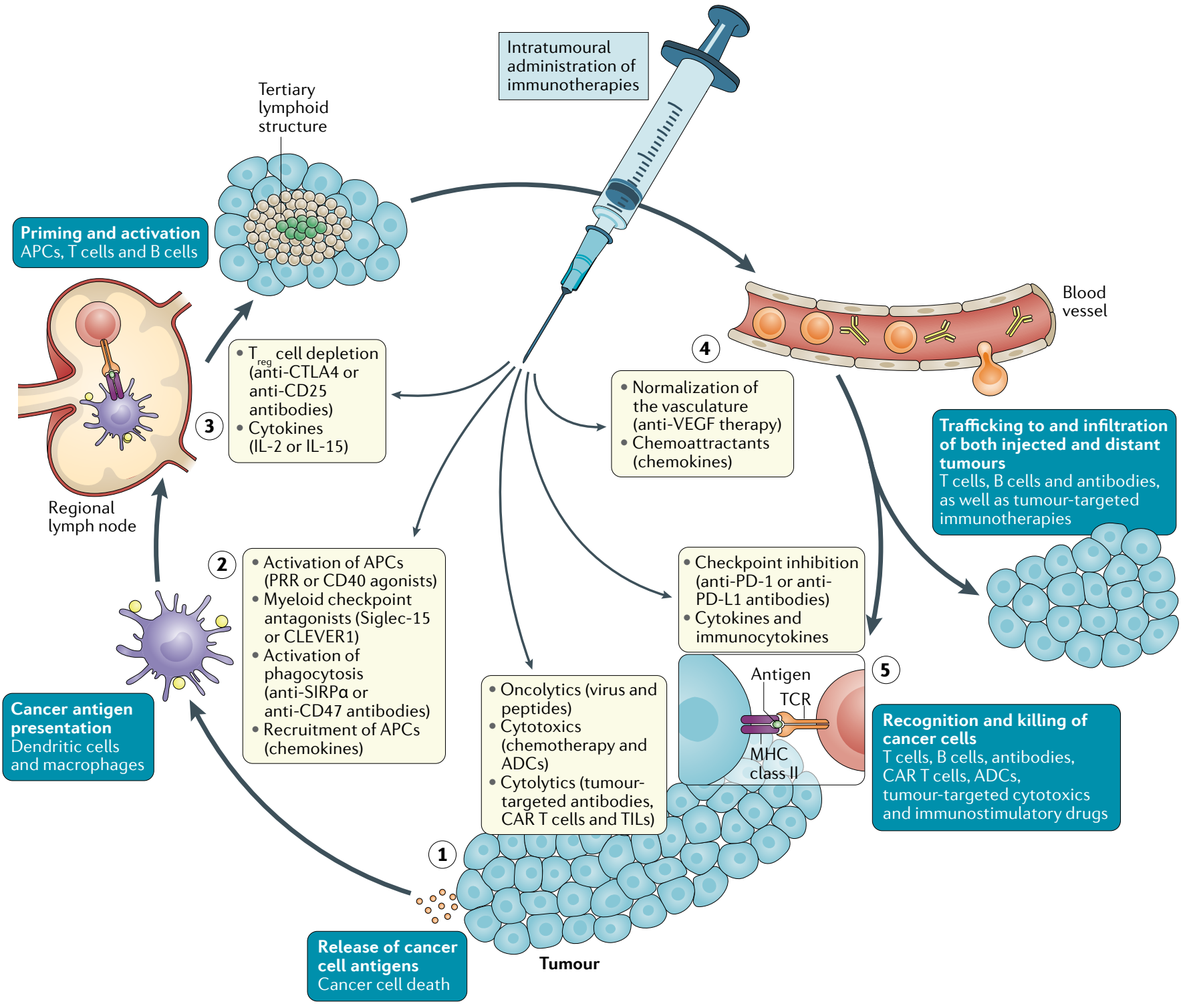

Fig. 3 | Boosting the intratumoural cancer immunity cycle. By selecting therapeutic agents based on their immunological properties, local immunotherapy - achieved either directly through intratumoural administration or indirectly through selective delivery to or activation in the tumour following systemic administration — can specifically enhance each step of the cancer immunity cycle described by Chen and Mellman ${ }^{31}$. Examples of key cell types, processes and immunotherapy agents that are relevant to each step of this cycle are noted in the figure. Importantly, in accordance with steps 4 and 5 of the cycle, local immunotherapies need to result in redistribution of effector immune cells or antibodies via the circulation for abscopal or anenestic responses against distant untreated lesions and micrometastases. ADCs, antibody-drug conjugates; APCs, antigen-presenting cells; CAR, chimeric antigen receptor; CLEVER1, common lymphatic endothelial and vascular endothelial receptor 1 (also known as stabilin 1); PRR, pattern recognition receptor; Siglec-15, sialic acidbinding Ig-like lectin 15; SIRPa, signal-regulatory protein- $\alpha$; TCR, T cell receptor, TILs, tumour-infiltrating lymphocytes; $\mathrm{T}_{\text {reg, }}$, regulatory $\mathrm{T}$.

Other TLR9 agonists include tilsotolimod, MGN1703 and CMP101, all of which are being tested as intratumoural immunotherapies in clinical trials (Supplementary Table 1). Tilsotolimod (previously known as IMO-2125) is the most advanced TLR9 agonist in clinical development and is being tested in combination with ipilimumab in a phase III trial involving patients with anti-PD-1 mAb-refractory advanced-stage melanoma (NCT03445533). This trial was initiated based upon promising results with the same combination in the phase I/II ILLUMINATE-204 trial (NCT02644967), which revealed an objective response rate (ORR) of $22 \%$, a disease control rate of $71 \%$ and a median overall survival (OS) duration of 21 months in this difficult-to-treat patient population ${ }^{46}$.

TLR4 agonists. TLR4 is a cell-surface receptor that recognizes bacterial lipopolysaccharides (LPS) and was discovered as a result of its roles in septic shock and DC maturation ${ }^{47,48}$. In addition to LPS, TLR4 responds 
to endogenous stimuli, such as the nuclear protein HMGB1, that act as damage-associated molecular patterns (DAMPs) ${ }^{49}$. Systemic targeting of TLR4 is clearly dangerous given that this receptor is the main mediator of septic shock; therefore, localized targeting is required.

A more defined and detoxified form of LPS, known as monophosphoryl lipid A (MPL), was developed as an adjuvant for intradermal vaccines and is currently used in combination with other adjuvants in vaccine development ${ }^{50}$. MPL has not been used clinically for intratumoural immunotherapy despite many reports showing antitumour effects of intratumoural injection of LPS in mouse xenograft models ${ }^{51}$.

The TLR4 agonist G100 is a fully synthetic analogue of LPS. This compound has been tested intratumourally in patients with Merkel cell carcinoma, with objective tumour regressions observed in both the neoadjuvant and metastatic settings ${ }^{52}$. G100 has also been tested in combination with low-dose irradiation, with or without pembrolizumab or rituximab, in a phase I/II trial involving patients with follicular lymphoma (NCT02501473); the approach was remarkably safe and the ORR with G100 plus irradiation was $26 \%{ }^{53}$. In this setting, consideration must be given to the fact that the malignant $B$ cells express functional TLR4 and are therefore likely to be directly responsive to G100. Indeed, responses tended to occur in patients with higher levels of expression of TLR4 on malignant cells ${ }^{54}$.

TLR3 agonists. TLR3 is an endosomal receptor for double-stranded RNAs (dsRNAs) that often constitute viral genomes or intermediates in the viral replication cycle $^{55}$. Polyinosinic:polycytidylic acid (poly I:C) is a self-hybridizing dsRNA analogue that was originally described as a potent inducer of IFN $\alpha / \beta$ production by a variety of leukocytes ${ }^{56}$. TLR3 is prominently expressed in DCs, including conventional type $1 \mathrm{DCs}(\mathrm{cDC} 1 \mathrm{~s})$ that are responsible for cross-presenting tumour-associated antigens to $\mathrm{CD}^{+} \mathrm{T}$ cells ${ }^{57}$. Interestingly, intratumoural injection of poly I:C induces potent immune-mediated tumour regression in mouse model ${ }^{58,59}$.

Three poly I:C-based molecules have entered clinical development: rintatolimod, Hiltonol and BO-112. The poly I:C moiety is stabilized with poly-L-lysine and carboxymethylcellulose in Hiltonol and through nanoplexing with polyethylenimine in BO-112. Most of the published experience with intratumoural administration of TLR3 agonists relates to Hiltonol ${ }^{60,61}$. This agent has been used as an adjuvant for several antigen-defined vaccines and induces IFN $\alpha / \beta$ responses in healthy volunteers when subcutaneously administered alone ${ }^{62}$. Hiltonol has also been used safely for intramuscular administration as an adjuvant for investigational glioblastoma vaccines ${ }^{63}$. Intratumoural injections of Hiltonol alone have been reported to result in tumour control and combinations with DC vaccines and radiotherapy have been associated with remarkable disease control in a small number of patients with metastatic solid tumours, with anenestic effects in non-treated lesions ${ }^{61}$; however, the absence of comparative randomized studies precludes conclusions on efficacy.
Intratumoural $\mathrm{BO}-112$ has antitumour activity in mouse models ${ }^{64}$. In addition to TLR3, BO-112 activates the cytoplasmic RGR family RNA helicases MDA-5 and RIG- ${ }^{65}$ and potentially also protein kinase R (PKR), which is another cytoplasmic PRR for dsRNA ${ }^{66}$. Notably, following intratumoural delivery, this compound kills a fraction of tumour cells through mechanisms that reportedly include intense autophagy ${ }^{65}$. Repeated intratumoural injection of BO-112 is safe in humans and induces a type I IFN transcriptional signature and $\mathrm{CD}^{+} \mathrm{T}$ cell infiltration into the injected lesions. Addition of BO-122 to anti-PD-1 mAbs was also well tolerated and resulted in objective responses in $3(11 \%)$ of 28 patients with primary resistance to anti-PD-1 mAbs ${ }^{67}$.

Activation of MDA- 5 and RIG-I by dsRNAs, such as BO-112, also induces potent type I IFN responses ${ }^{68}$. To elicit this pathway, however, some degree of penetration of the dsRNA analogues into the cytoplasm is required $^{69}$. The importance of dsRNA recognition in the cytosol is underscored by the preclinical findings that tumours lacking the RNA-editing enzyme ADAR1 accumulate cytosolic dsRNAs and are highly sensitive to anti-PD-1 $\mathrm{mAbs}^{70}$; MDA-5 and PKR are implicated in this sensitization ${ }^{70,71}$.

TLR7 and TLR8 agonists. TLR7 and TLR8 are endosomal receptors that recognize single-strand RNAs (ssRNAs) with viral features, such as abundant GU dinucleotide motifs ${ }^{72,73}$. Pharmacological compounds of the imidazoquinoline family, which are proinflammatory irritants, were discovered to be agonists of these receptors ${ }^{74,75}$. Such compounds include imiquimod and resiquimod. Imiquimod (formulated as a $5 \%$ topical cream) is widely used in dermatology for the treatment of genital warts ${ }^{76}$. In addition, repeated local application of the cream to superficial basal cell carcinoma (BCC) lesions results in responses in most patients and can be curative or facilitate subsequent more-conservative surgery ${ }^{77}$, and this approach gained FDA approval in 2004. An intense inflammatory infiltrate of pDCs is a key effector mechanism ${ }^{77}$, although NK cells, T cells and IFNs are also likely to be involved. Encouraged by these results, Dutch investigators used the cream to treat women with vulvar intraepithelial neoplasia, a human papillomavirus (HPV)-related premalignant condition, with a complete response (CR) rate of $>80 \%$ and good tolerability ${ }^{78}$. Furthermore, imiquimod has been tested as a treatment for cutaneous metastasis of breast cancer in combination with radiotherapy, enhancing the local response to radiotherapy from $11 \%$ to $66 \%{ }^{79}$. Topical use of resiquimod in a gel formulation has clinical activity against cutaneous $\mathrm{T}$ cell lymphoma ${ }^{80}$. However, intratumoural injection of TLR7/8 agonists remains to be explored clinically.

cGAS/STING agonists. The presence of cytosolic dsDNA is indicative of viral or bacterial infection or severe tissue damage, and can be detected through a variety of mechanisms. These mechanisms include allosteric binding of the dsDNA to the nucleotide cyclase enzyme cGAS, which subsequently synthesizes cyclic 
guanosine monophosphate-adenosine monophosphate (cGAMP) dinucleotides ${ }^{81}$. In turn, cGAMP (or its bacterial counterparts) activate STING that is deployed on the cytosolic surfaces of Golgi and cytoplasmic vesicles to elicit potent type I IFN transcription through the TBK1-IRF3 signalling pathway ${ }^{74-76}$. The potency of this defence mechanism is emphasized by the fact that preventing cytosolic dsDNA degradation via genetic deletion of the cytosolic DNAse Trex1 results in fatal systemic inflammation and autoimmunity in juvenile mice $^{82}$. An endogenous role for the cGAS-STING pathway in antitumour immunity has been uncovered, whereby $\mathrm{cDC} 1 \mathrm{~s}$ foster antigen cross-presentation to $\mathrm{CD}^{+} \mathrm{T}$ cells and productive co-stimulation of cytotoxic $\mathrm{T}$ cell responses ${ }^{83,84}$. Moreover, cGAS-STING signalling also seems to be important for the immunostimulatory effects of radiotherapy, including abscopal responses when combined with immune-checkpoint inhibition $^{85}$.

The most advanced method of exploiting the cGASSTING pathway involves the use of various cyclic dinucleotides optimized for binding to human STING isoforms (five haplotypes of STING1 are known). In mice, intratumoural injection of the cyclic dinucleotide vadimezan (also known as DMXAA) promotes antitumour immunity, including systemic immunity against non-injected tumours, which can be further enhanced by concomitant PD-1 inhibition ${ }^{86}$. A number of clinical trials have investigated intravenous vadimezan, but have shown disappointing anticancer activity ${ }^{87}$, although this agent is a poor agonist of most STING isoforms ${ }^{86}$. Other intratumourally administered cyclic dinucleotide agonists of human STING have entered clinical trials (Supplementary Table 1), with good safety but minimal antitumour activity observed to date with monotherapy, even upon repeated injection ${ }^{88,89}$.

Studies of local STING agonists in combination with systemic anti-PD-1 mAbs are also ongoing (Supplementary Table 1). In a phase I trial involving 66 patients with solid tumours, repeated intratumoural injection of the STING agonist MIW815 (ADU-S100) in combination with the anti-PD-1 mAb spartalizumab was safe and induced objective responses in a minority of patients with triple-negative breast cancer or anti-PD-1 mAb-resistant melanoma ${ }^{89}$. Similarly, in a phase I trial, another intratumoural STING agonist, MK-1454, in combination with pembrolizumab had good tolerability but limited clinical activity ${ }^{88}$.

Thus, the high expectations surrounding STING agonists have not been satisfied so far. However, novel STING agonists with potentially different mechanisms of action are under clinical development, including E7766 for both intratumoural immunotherapy of lymphoma and solid tumours (NCT04144140). Intratumoural administration of another novel STING agonist, BMS-986301, is being tested in combination with nivolumab plus ipilimumab in patients with various solid tumours (NCT03956680) ${ }^{90}$. Other STING agonists are currently being developed for intravenous rather than intratumoural delivery, including GSK3745417 (with or without pembrolizumab; NCT03843359 and NCT03010176) ${ }^{90}$.
Bacterial toxins as poly-PRR agonists. Besides intravesical BCG, another type of local bacterial immunotherapy is still used as a standard therapy in oncology. This bacterial therapy, OK-432, comprises a lyophilized mixture of group A S. pyogenes strains and therefore closely reflects Coley toxins. OK-432 is currently approved in Japan and Taiwan and is available in the USA for the treatment of lymphangioma, supported by multiple reports that intratumoural injection induces ORRs of $>80 \%$ in patients with such cystic lesions ${ }^{91,92}$.

Beyond lymphangioma, a retrospective study of 26 patients with advanced-stage ovarian cancer treated with intraperitoneal OK-432, IL-2 and platinum plus paclitaxel chemotherapy revealed a total recurrence rate of $53.8 \%$, compared with $88.0 \%$ in 25 equivalent patients treated with standard platinum plus paclitaxel chemotherapy $(P=0.0128)^{9}$. At 1 month after treatment, white blood cell, absolute neutrophil and absolute lymphocyte counts were all significantly higher in patients treated with immunochemotherapy $(P<0.05$ for each comparison). In patients with malignant pleural effusions of NSCLC, a randomized phase II trial designed to determine the optimal dose of OK-432 for intrathoracic administration revealed a lesion control rate at day 8 of $79 \%$ with a dose of 10 Klinische Einheit (KE) and of 53\% with a dose of $1 \mathrm{KE}^{93}$. In a retrospective analysis of data from 16 patients with metastatic colorectal cancer and malignant effusions (13 with ascites and 3 with pleural effusions), locoregional administration of OK-432 alone $(0.2-5 \mathrm{KE})$ or in combination with IL-2 (100,000 IU) at the time of paracentesis resulted in prolonged disappearance of the effusion in 7 (64\%) of 11 and 4 (80\%) of 5 patients, respectively ${ }^{94}$.

The pro-inflammatory properties of OK-432, which has intrinsic agonistic activity for TLR2, TLR4 and TLR9, are reliant on effects on both $\mathrm{CD} 4^{+} \mathrm{T}$ cells and macrophage infiltration ${ }^{94}$; this agent has also been shown to decrease the number and immunosuppressive effects of regulatory $\mathrm{T}\left(\mathrm{T}_{\text {reg }}\right)$ cells in an IL-12-dependant manner ${ }^{95}$.

Despite the promising activity of OK-432, studies of this product in combination with immune-checkpoint inhibitors are lacking. Of note, co-injection of several chemically distinct PAMPs into a tumour might synergize in potentiating antitumour immunity (M.A., unpublished observations).

\section{Immunogenic oncocytotoxic agents}

Oncolytic viruses. Oncolytic viruses preferentially infect and kill cancer cells over non-malignant cells. The first FDA and EMA approved oncolytic virus, talimogene laherparepvec (T-VEC), is a form of herpes simplex virus-1 (HSV-1) genetically modified to reduce its pathogenicity and to encode human GM-CSF. Intratumoural injection of T-VEC is approved for the treatment of superficial melanoma metastases in patients with stage IIIB-IVM1a (EMA) or stage IIIB-IVM1c melanoma (FDA). These approvals were based on data from the phase III OPTiM trial, which demonstrated a higher rate of durable responses lasting $\geq 6$ months with T-VEC versus subcutaneous GM-CSF (19.0\% versus $1.4 \%$; OR $16.6,95 \%$ CI $4.0-69.2 ; P<0.0001)$, as well as superior OS 
(median 23.3 months versus 18.9 months; HR 0.79, $95 \%$ CI $0.62-1.00 ; P=0.049)^{96}$. The median time to response with T-VEC was 4.1 months ${ }^{97}$, which is substantially longer than that observed with systemic anti-PD-1 mAbs (for example, 2.8 months with pembrolizumab in patients with advanced-stage melanoma $)^{98}$. Moreover, 23 (48\%) of 48 patients with durable responses had transient disease progression before responding, including 14 patients who developed new lesions (that is, not pseudoprogression of pre-existing lesions ${ }^{99}$. Interestingly, no difference in OS was found in patients with disease progression prior to response versus those without progression, and the median duration of response was not reached in the former group ${ }^{99}$. Overall, 995 (47\%) of 2,116 injected lesions as well as $212(22 \%)$ of 981 uninjected non-visceral lesions and 16 (9\%) of 177 uninjected visceral lesions resolved completely in patients who received T-VEC ${ }^{99}$. The activity of intratumoural T-VEC against non-injected lesions was higher if the uninjected lesions were in the same anatomical region as the injected lesions. Indeed, the CR rate was 36\% (107 of 294) for uninjected non-visceral lesions located at the same site as an injected lesion, compared with 13\% (39 of 306) for those at different sites ${ }^{99}$. Pre-existing immunity against HSV-1 did not seem to hamper the efficacy of T-VEC ${ }^{96}$. Of note, however, the GM-CSF control treatment used in OPTiM has limited or no efficacy in patients with melanoma ${ }^{100}$. Importantly, combinations of T-VEC with anti-CTLA4 and anti-PD-1 mAbs have not raised safety concerns in patients with melanoma and have promising antitumour activity ${ }^{101,102}$. Whether such combinations have efficacy superior to that of anti-PD-1 mAb monotherapy in patients with stage IIIB-IVM1c melanoma is currently being tested in a phase III trial (NCT02263508; Supplementary Table 2). New herpesvirus-based vectors encoding GM-CSF, some armed with additional immune-enhancing transgenes (for example, encoding CD40L or 4-1BBL), are currently being developed for intratumoural administration. Early evidence of biological and clinical

\section{Box 1 | Intratumoural immunotherapy with antiviral vaccines}

We and others have hypothesized that attenuated vaccines against infectious agents could be used as a source of pathogen-associated molecular patterns (PAMPs) and potential oncolytic viruses for intratumoural immunotherapy ${ }^{242}$. Indeed, vaccines against influenza, yellow fever and rotavirus can stimulate immune responses owing to their proinflammatory PAMPs and/or can induce cancer cell lysis to release tumour antigens ${ }^{242}$. Thus, such vaccines might activate antitumour immunity following intratumoural delivery. Indeed, in mouse models, intratumoural delivery of unadjuvanted influenza vaccines can turn immunologically 'cold' tumours 'hot' by increasing the number of $C D 8^{+} T$ cells and decreasing the levels of regulatory B cells in the tumour microenvironment ${ }^{243}$. In addition, intratumoural rotavirus vaccines can overcome the resistance of tumours to anti-CTLA4 and anti-PD-L1 antibodies ${ }^{244}$. Moreover, when using commercial rotavirus or yellow fever vaccines, previous systemic immunization with the live virus vaccines did not hamper the efficacy of subsequent intratumoural administrations ${ }^{244,245}$. Interestingly, the oncolytic properties of rotavirus were dispensable when used in combination with anti-PD-L1 antibodies; only the agonistic properties of viral nucleic acids on the RNA helicase pattern recognition receptor RIG-I seemed to be required for synergistic efficacy ${ }^{244}$. The repurposing of anti-infection vaccines as intratumoural immunotherapies could be facilitated by the fact that these products are GMP grade and commercially available. However, several practical questions must be addressed to achieve clinical translation, including the optimal vaccine dose, volume and schedule as well as the ideal target tumours. activity has been obtained for the GM-CSF-encoding herpesvirus RP1 (REF. $\left.{ }^{103}\right)$.

Pexastimogene devacirepvec (Pexa-Vec) is the second most advanced oncolytic virus in clinical development. Similarly to T-VEC, this vaccinia poxvirus has been genetically modified to encode GM-CSF. Development of this agent has mostly been focused on hepatocellular carcinoma (HCC), with clinical activity observed against injected and non-injected tumours through the induction of both cellular and humoral immune responses ${ }^{104,105}$. Intratumoural Pexa-Vec is currently being tested in early phase trials in combination with intratumoural ipilimumab (NCT02977156) as well as with intravenous anti-CTLA4 or anti-PD-1 mAbs (Supplementary Table 2). However, results of the phase III PHOCUS trial of intratumoural Pexa-Vec plus sorafenib versus sorafenib alone (NCT02562755) have been reported to be negative $\mathrm{e}^{106}$.

Other strains of oncolytic viruses have been shown to enhance the antitumour activity of immunostimulatory $\mathrm{mAbs}$ in preclinical studies ${ }^{107}$ and in early phase clinical trials $^{108}$. Moreover, intratumoural administration of the oncolytic adenoviruses DNX-2401 (REF. ${ }^{109}$ ) and teserpaturev produced promising results in patients with glioblastoma ${ }^{110}$.

Next-generation 'armed' oncolytic viruses modified to encode other immunostimulatory cytokines, co-stimulatory ligands or mAb immune-checkpoint inhibitors are being developed, with the goal of in situ combination immunotherapy following either systemic or intratumoural administration (Supplementary Table 2). In mice, intratumoural injections of an oncolytic vaccinia virus encoding IL-7 and IL-12 can convert poorly immunogenic tumours into inflamed tumours and induce complete regressions, even at distant non-injected tumour sites ${ }^{11}$. Indeed, oncolytic viruses are a versatile platform for combining various immunomodulatory agents into a single immunotherapy product. Moreover, efforts to repurpose approved attenuated viral vaccines for intratumoural immunotherapy are ongoing (BOX 1).

Oncolytic molecules. Beyond oncolytic viruses, several other agents have oncolytic properties and induce immunogenic cell death (ICD), but without the clinical hurdles associated with the use of pathogenic and/or genetically modified organisms ${ }^{112}$. For instance, considerable experience is available with intratumoural chemotherapy, which is an approach that has not yet been properly combined with immune-checkpoint inhibition (BOX 2).

Intratumoural immunotherapy with PV-10, a water-soluble derivative of the xanthene dye rose Bengal, has been advanced to clinical stages of testing (Supplementary Table 3), following promising results in bilateral tumour models in mice (in which only the tumours on one side of the body are treated but antitumour activity is often observed in both lesions) $)^{113,114}$. PV-10 has a mechanism of action that involves ICD, and disruption of lysosomes owing to accumulation of the dye is correlated with tumour-specific immune responses $^{113,114}$. A phase II trial of intratumoural PV-10 


\section{Box 2 | Intratumoural chemotherapy}

Several chemotherapies have been shown to induce immunogenic cell death (ICD), a form of cell death that can increase the immune recognition of cancer cells ${ }^{246,247}$. Examples of common chemotherapies with this capacity include cyclophosphamide, doxorubicin, mitoxantrone and oxaliplatin ${ }^{248}$. This rationale currently supports the combination of immune checkpoint-targeted antibodies with systemic chemotherapies. However, the approved chemotherapy-immunotherapy combinations currently indicated for non-small-cell lung cancer (NSCLC), small-cell lung cancer, triple-negative breast cancer and head and neck squamous cell carcinoma involve conventional high-dose chemotherapy regimens, which were historically selected based on the maximum tolerated dose, at which the main toxicities are cytopenias, including lymphopenia that is likely to reflect depletion of antitumour lymphocytes. Unsurprisingly, therefore, the median duration of tumour responses with such combinations are not very different from those observed with chemotherapy alone in all of the registration trials ${ }^{249,250}$. Indeed, data from longer-term follow-up studies of those trials ${ }^{251}$, preclinical data ${ }^{252}$ and case reports ${ }^{253}$ suggest that systemic high-dose chemotherapies have a detrimental effect on the efficacy of anti-PD-1 and anti-PD-L1 antibodies, thus supporting the idea that metronomic chemotherapy might be a better way to exploit these immunomodulatory effects.

Most of the in vivo preclinical rationale for ICD has been generated with intratumoural delivery of chemotherapies ${ }^{254,255}$, and the clinical translation of this strategy, especially in the context of immunotherapy, has been limited. Intratumoural cisplatin has been tested in a few clinical trials. For example, in a study of a cisplatin and epinephrine gel involving 25 patients with relapsed and/or refractory melanoma ${ }^{256}$, the objective response rate for all evaluable injected cutaneous or soft-tissue metastases was $53 \%$ ( 130 of 244 , including 114 complete responses). CT-guided intratumoural administration of a cisplatin and epinephrine gel was also tested in eight patients with a total of 17 colorectal liver metastasis and in nine patients with 13 hepatocellular carcinoma nodule ${ }^{257}$, and resulted in 6-month local control rates of $38 \%$ and $71 \%$, respectively. Intratumoural delivery of cisplatin by endobronchial ultrasonography-guided transbronchial needle injection has also been reported to be safe and have good antitumour activity in patients with peribronchial NSCLC ${ }^{258}$. Importantly, local administration permits a drastic reduction in the chemotherapy doses ${ }^{259}$. Beyond cisplatin, other chemotherapies have been injected directly into NSCLC tumours, including 5 -fluorouracil, mitomycin, methotrexate, bleomycin and mitoxantrone ${ }^{260}$. Beyond conventional cytotoxic chemotherapies, new families of chemical products capable of inducing ICD are currently being developed for intratumoural administration (Supplementary Table 3). To our knowledge, intratumoural chemotherapies in combination with systemic (or local) immune-checkpoint inhibition have never been tested, but might leverage the benefits of ICD while preserving antitumour effector $T$ cells and $B$ cells. in patients with in-transit metastatic melanoma produced a promising ORR of $87 \%^{115}$. This treatment has also been combined with radiotherapy for patients with metastatic melanoma, resulting in an ORR of $>85 \%{ }^{116}$. On the basis on these results, intratumoural PV-10 has received orphan drug designation from the FDA. Ongoing clinical studies of PV-10 include a phase III trial in patients with locally advanced $B R A F$ wild-type cutaneous melanoma that has progressed on immune-checkpoint inhibitors (NCT02288897), as well as phase II studies involving patients with HCC or liver metastasis (NCT00986661), or those with melanoma, in combination with systemic pembrolizumab (NCT02557321) (Supplementary Table 3). Indeed, preclinical evidence suggests synergistic effects with PD-1 blockade ${ }^{117}$. Intratumoural immunotherapy with PV-10 might warrant screens for other chemical compounds that can elicit ICD and delayed type hypersensitivity reactions inside injected tumours.

Tigilanol tiglate is another novel molecule of interest for intratumoural immunotherapy. This compound is not synthetically tractable but is isolated from the seeds of Fontainea picrosperma (the blushwood tree). Tigilanol tiglate has been approved in Europe for the treatment of canine mast cell tumours by intratumoural administration, based on a CR rate of $88 \%$ in a randomized controlled trial in $\operatorname{dog} \mathrm{s}^{118}$, and has also demonstrated activity against human cancers. In a first-in-human (FIH) phase I study, the safety and activity of intratumoural tigilanol tiglate was evaluated in 22 patients with solid tumours. The maximum tolerated dose (MTD) was not reached and treatment was generally well tolerated. Injection site reactions occurred in all patients, even at the lowest doses. Six patients (27\%) had an objective response, with four RECIST-defined CRs, including in patients with skin squamous cell carcinoma (SCC), melanoma or angiosarcoma ${ }^{119}$. Tigilanol tiglate, perhaps in addition to inducing ICD, acts as a protein kinase $\mathrm{C}(\mathrm{PKC})$ activator $^{120}$ and might, therefore, have multiple functional effects on immune cells, given that various PKC isoforms have crucial roles at the immune synapse and on $\mathrm{T}$ cell function and survival ${ }^{121-124}$.

Oncolytic peptides are another interesting class of intratumoural immunotherapies. These agents are derived from natural antimicrobial peptides but can also have activity against cancer cells ${ }^{125}$. For example, ruxotemitide is a cationic amphipathic peptide that can permeabilize mitochondrial membranes, thereby causing caspase-independent necrosis that is partially mediated by BAX and/or BAK1 (REFS ${ }^{113,114}$ ). Necrosis causes the release of DAMPs and is, therefore, a form of ICD $^{126,127}$. Preclinical data indicate that intratumoural ruxotemitide enhances tumour infiltration by activated $\mathrm{CD} 8^{+} \mathrm{T}$ cells and $\mathrm{CD} 4^{+} \mathrm{T}$ helper type $1\left(\mathrm{~T}_{\mathrm{H}} 1\right)$ cell responses, and induces systemic anticancer immunity against both injected and non-injected tumours ${ }^{128}$. A FIH phase I trial of intratumoural ruxotemitide as monotherapy in patients with several tumour types has revealed some clinical activity against sarcoma, in association with increased numbers of tumour-infiltrating lymphocytes (TILs) and clonal expansion of T cells ${ }^{128}$. Ruxotemitide is currently being tested in patients with advanced-stage solid tumours, including in combination with systemic pembrolizumab (NCT04796194). This agent is also being explored as a neoadjuvant therapy to recruit $\mathrm{T}$ cells to the tumours prior to TIL isolation and expansion for adoptive cell therapy in patients with soft-tissue sarcoma (NCT03725605).

\section{Local administration of cytokines}

Data from preclinical models provide a strong rationale for intratumoural administration of cytokines in combination with immunostimulatory mAbs ${ }^{129,130}$. Systemic (intravenous or subcutaneous) therapy with cytokines, predominantly with IL-2, IFNa or TNF, but also with IL-7 or IL-15 (REFS ${ }^{131,132}$ ), has been tested extensively in patients with cancer, with the aim of expanding pre-existing antitumour immune responses. In general, this systemic strategy had limited efficacy and was associated with severe adverse events, suggesting a narrow therapeutic window, and has mostly been abandoned; however, this approach is currently being revamped using new agents and engineered cytokine constructs, with the aim of enhancing the efficacy of other immunotherapies, including immune-checkpoint inhibitors ${ }^{133,134}$. 
Notwithstanding, the main targets of these cytokines are tumour-infiltrating immune cells, and thus local delivery would be expected to increase the therapeutic index, and is being explored in several clinical trials using various cytokines (Supplementary Table 4).

IL-2. Aldesleukin, a recombinant form of human IL-2, was first approved by the FDA for the treatment of metastatic renal cell carcinoma (RCC) in 1992, and subsequently for melanoma in 1998. However, clinical use of IL-2 has been limited owing to its short half-life (which requires frequent administrations of high doses), frequent treatment failure (possibly related to the activation of $\mathrm{T}_{\text {reg }}$ cells, which express high levels of the high-affinity IL-2 receptor (IL-2R) complex containing the a subunit, also known as CD25), and its common dose-limiting toxicities (DLTs; such as capillary leak syndromes and multiorgan toxicities $)^{135}$.

Direct intratumoural delivery might mitigate the toxicities of systemic cytokine therapy whilst also maximizing local bioavailability and activity ${ }^{136}$. Intralesional IL-2 has been tested broadly in the setting of melanoma, resulting in ORRs of up to $50 \%$ in patients with in-transit cutaneous or subcutaneous metastases ${ }^{137}$. However, the ORR was much lower $(\sim 10 \%)$ for deep-seated metastases ${ }^{138}$. This difference in ORRs between superficial and visceral metastasis is not fully understood. Combinatorial approaches are of interest to address the potential issue of $\mathrm{T}_{\text {reg }}$ cell expansion by IL-2 (for example, combination with anti-CTLA4 mAbs, as discussed later in this Review).

New forms of IL-2 molecularly engineered to have lower affinity for CD25 and to preferentially stimulate the low-affinity IL-2R complex containing only the $\beta$ and $\gamma$ subunits (CD122 and CD132, respectively), with or without a longer half-life in vivo, are currently in clinical development. For example, bempegaldesleukin is a pegylated form of aldesleukin ${ }^{139,140}$ that has promising safety and clinical activity in patients with solid tumours following systemic administration in combination with nivolumab (ORR 59.5\%, CR rate 18.9\%) $)^{134}$. Of note, systemic bempegaldesleukin (with or without nivolumab) is currently being tested in combination with the intratumoural pegylated TLR7/8 agonist NKT-262 (NCT03435640) (Supplementary Table 1). Intratumoural administration of bempegaldesleukin might further increase local activation of TILs.

IFN $\gamma$. Intratumoural IFNs have been broadly investigated in the clinic, but only in small cohorts. Intratumoural IFN $\gamma$ has been safely administered to nine patients with metastatic melanoma, at a dose of $2 \times 10^{6} \mathrm{IU}$ and together with MHC I-restricted and MHC II-restricted melanoma peptide vaccines; when administered 22 days after the first vaccine dose, IFN $\gamma$ increased the production of CXCL10, CXCL11 and CCL5, but failed to promote immune cell infiltration or induce an antitumour immune gene signature ${ }^{141}$.

IFNa and IFN $\beta$. Intralesional IFNa-2a $\left(1-3 \times 10^{6} \mathrm{IU}\right.$ per injection) is widely used for the local treatment of keratoacanthoma, including large lesions $(>2 \mathrm{~cm})$, and can spare patients from local chemotherapy, surgery or radiotherapy ${ }^{142}$. Many clinical reports support the therapeutic value of intralesional IFNa or IFN $\beta$ for BCC and cutaneous SCC ${ }^{143-145}$. However, IFNa-2b $\left(\sim 1.5 \times 10^{6}\right.$ IU per injection) seems to have limited efficacy against lesions $>2 \mathrm{~cm}$ in diameter ${ }^{143}$. Interestingly, a proposed mechanism of action involves the FAS-FAS ligand pathway ${ }^{146}$. Indeed, BCC cells constitutively express FAS ligand but not FAS and can, therefore, evade local antitumour immune responses by inducing FAS-mediated apoptosis of $\mathrm{FAS}^{+} \mathrm{CD} 4^{+} \mathrm{T}$ cells ${ }^{146}$. IFNa can circumvent this immune escape mechanism by upregulating FAS on BCC cells ${ }^{146}$. Most research with type I IFNs has focused on inducing tumour $\mathrm{T}$ cell infiltration, although preclinical data suggest that the therapeutic effects of IFN $\beta$ could be related to its anti-angiogenic properties ${ }^{147}$.

$I L-15$. The biology of IL-15 is complex. IL-15 associates with IL-15Ra intracellularly and is then shuttled to the cell surface bound to this receptor subunit in order to stimulate adjacent cells expressing the IL-15R $\beta / \gamma$ complex - a mechanism known as 'trans-presentation. Moreover, IL-15Ra-IL-15 can be cleaved from the cell surface, generating transient but marked increases in the levels of soluble IL-15R $\alpha$-IL-15 complexes (sIL-15) in response to numerous immune stimuli, including total body irradiation, viral infections, TLR activation, CD40 stimulation, type I IFNs and STING signalling ${ }^{148}$. In mouse models, intratumoural expression of IL-15 via electroporation of plasmid DNA vectors has therapeutic activity against aggressive B16 melanomas (37.5\% of mice were alive with complete tumour regression at day 100 versus $0 \%$ of those receiving a control plasmid vector) ${ }^{149}$. Limited data have been generated on the value of intratumoural IL-15 in combination with other anticancer therapies. Several 'IL-15 superagonist' constructs, in which IL-15 is crosslinked to IL-15R $\alpha$, are now in clinical development with the aim of achieving greater and longerlasting IL-15 activity in vivo ${ }^{150}$; however, the clinical value of intratumoural IL-15 has not yet been tested.

IL-12. Intravenous or subcutaneous injections of recombinant human IL-12 have proved to be relatively safe and to generate objective responses, albeit with a narrow therapeutic window in early phase trials ${ }^{151-156}$. Currently, the most advanced form of IL-12-based intratumoural immunotherapy involves electroporation of tumours with tavokinogene telseplasmid (Tavo), a DNA plasmid encoding both the $\alpha$ (p35) and the $\beta$ (p40) subunits of the heterodimeric human IL-12 protein (Supplementary Table 4). This approach has been shown to induce the regression of both treated and distant lesions in patients with melanoma or Merkel cell carcinoma ${ }^{157,158}$. A lipid nanoparticle formulation of mRNA encoding singlechain IL-12 for intratumoural injection (MEDI1191) has also entered clinical testing in combination with systemic treatment with the anti-PD-L1 mAb durvalumab (NCT03946800), following excellent results in mouse models ${ }^{159}$. Preliminary evidence of activity has been reported, including two partial responses among ten patients with melanoma, including one with documented effects in non-injected lesions ${ }^{160}$. A similar 
approach involving ionizable cationic lipid nanoparticles containing self-replicating Venezuelan equine encephalitis alphavirus-based RNA constructs encoding singlechain IL-12 eradicated large established tumours and induced the regression of distal uninjected tumours in several mouse models ${ }^{161}$.

Combinations of multiple cytokines. Combinations of cytokines could be synergistic in their ability to stimulate antitumour immunity. Notably, intratumoural recombinant human IFNa-2a (rhIFNa-2a) has increased efficacy when used in combination with intravenous vincristine. In a veterinary study in dogs with canine transmissible venereal tumour, concurrent or sequential administration of rhIFNa-2a intratumourally and vincristine intravenously led to substantially shorter durations of therapy required for a CR than vincristine chemotherapy alone (mean 3.17 weeks and 3.50 weeks for concurrent and sequential therapy, respectively, versus 5.11 weeks for vincristine alone) ${ }^{162}$. The combination of intratumoural IFN $\alpha-2 b$ and IL- 2 was tested in ten patients with cystic glioblastoma without any peritumoural oedema (which was anticipated a priori) or other safety issues; however, weekly injections for 4 weeks had no effect on tumour progression nor OS in these patients ${ }^{163}$.

Alternative ways of delivering combinations of cytokines to achieve sustained in situ exposure of these short-lived proteins are gaining traction. One approach involves anchoring the cytokines to collagen to substantially prolong retention in the tumour bed following intratumoural injection. This strategy has been associated with synergistic antitumour activity with combinations of IL-2 and IL-12 in several mouse models, while limiting systemic exposure ${ }^{164}$. An alternative strategy involves intratumoural injection of cocktails of cytokine-encoding mRNAs ${ }^{165}$. For example, the triple combination of IL-23, IL-36 $\gamma$ and OX40L mRNAs overcomes the resistance of aggressive tumours to single or dual intratumoural cytokine therapy in preclinical models ${ }^{166}$. Such intratumoural cytokine mRNA-based therapies are currently under active clinical development (NCT03739931 and NCT03871348), and preliminary evidence of good tolerability and immunological activity have been obtained with an OX40L-encoding mRNA ${ }^{167}$.

\section{Immunostimulatory monoclonal antibodies}

No clear relationships among dose, clinical efficacy and toxicities have been established for anti-PD-1 or anti-PD-L1 mAbs. Indeed, the mechanism of action of these agents relies on a purely antagonistic rationale, and no additional safety or efficacy signals are evident at doses beyond those that result in saturation of the target ${ }^{168,169}$. With a number of other immunostimulatory mAbs, however, DLTs prevent use of the optimal therapeutic dose. Intratumoural delivery of such immunostimulatory mAbs might, therefore, increase the therapeutic index while limiting systemic exposure and associated immune-related adverse events (irAEs). A variation of this approach in which immune-checkpoint inhibitors are delivered into tumour-draining lymph nodes has been reported to have local and distant antitumour effects in mice ${ }^{170}$.
As opposed to anti-PD-1 or anti-PD-L1 mAbs, the only approved anti-CTLA4 mAb, ipilimumab, has greater clinical efficacy at higher doses when used alone $^{171}$ or in combination with anti-PD-1 mAbs ${ }^{172}$. Evidence from preclinical and clinical studies indicates that the efficacy of anti-CTLA4 mAbs is dependent on $\mathrm{F} c \gamma$ receptor-mediated depletion of intratumoural $\mathrm{T}_{\text {reg }}$ cells (which express high levels of surface CTLA4) through antibody-dependent cellular cytotoxicity (ADCC) $)^{171-174}$. The same mechanism of action is likely to result in depletion of $\mathrm{T}_{\text {reg }}$ cells from non-malignant tissues, which might explain the irAEs induced by ipilimumab, although no data are currently available to support this conclusion. Nevertheless, intratumoural delivery of anti-CTLA4 mAbs could enable the use of optimal doses while preventing toxicities associated with systemic exposure ${ }^{173}$; this approach has been shown to have potent antitumour activity in multiple mouse models ${ }^{174-177}$. In models of tumours refractory to intralesional anti-CTLA4 mAbs, intratumoural combinations with TLR9 agonists can overcome resistance and generate systemic antitumour immune responses ${ }^{178}$. Clinical translation of intratumoural anti-CTLA4 therapy is underway (Supplementary Table 5). For example, in a phase I study of intratumoural co-injection of ipilimumab $(0.5,1$ or $2 \mathrm{mg})$ and IL- $2\left(3 \times 10^{6} \mathrm{IU}\right)$ in 12 patients with unresectable melanoma ${ }^{179}$, no DLT was observed, seven patients (58\%) had a CR at injected lesions and three (30\%) of ten evaluable patients had a partial response as per immune-related response criteria (irRC); eight (89\%) of nine patients with more than one tumour had locoregional or distant abscopal responses. This combination is based on the rationale of enhancing T cell function with IL-2 whilst mitigating counterproductive effects of this cytokine on $\mathrm{T}_{\text {reg }}$ cells through anti-CTLA4 mAb-mediated depletion of this immunosuppressive cell type. These results should be interpreted with caution, however, given the limited number of patients. Another ongoing clinical trial has provided preliminary evidence that injection of a combination of anti-CTLA4 and anti-PD-1 mAbs directly into sites of glioblastoma resection is safe ${ }^{180}$. Several other clinical trials are currently evaluating the safety and efficacy of intratumoural anti-CTLA4 mAbs in combination with other therapies (Supplementary Table 5).

No specific DLTs have been identified for agonistic anti-OX40 mAbs in clinical studies ${ }^{181,182}$; however, the antitumour activity of these agents in syngeneic mouse models also relies on Fc $\gamma$ R-dependent depletion of intratumoural $\mathrm{T}_{\text {reg }}$ cells ${ }^{183,184}$. Thus, systemic use of co-stimulatory agonist $\mathrm{mAbs}$ raises concerns of immune overactivation, an effect dramatically observed in the clinic with a superagonist anti-CD28 $\mathrm{mAb}^{185}$. Intratumoural administration of anti-OX40 mAbs has been tested in several mouse models, revealing synergistic activity in combination with TLR9 agonists ${ }^{178,186}$. This approach is currently being explored in clinical trials (for example, NCT03410901; Supplementary Table 1).

Beyond anti-CTLA4, other immunostimulatory $\mathrm{mAbs}$ have evidence of relationships between dose and efficacy and/or toxicity. For example, hepatotoxicity 
has been observed as a DLT upon intravenous administration of the agonistic anti-CD137 mAb urelumab; therefore, intratumoural administration of urelumab at the MTD is being tested in combination with systemic nivolumab in an ongoing trial (NCT03792724). The anti-CD40 mAb selicrelumab has also been associated with DLTs upon intravenous administration ${ }^{187}$. With the same rationale of increasing the therapeutic index, intratumoural administration of agonistic mAbs targeting CD137 or CD40 has been tested in syngeneic mouse models, mostly in combination with other immunotherapies ${ }^{188-193}$. In xenograft models, this intratumoural strategy was associated with activity against both injected and distant tumours ${ }^{188-193}$. Several trials are investigating this approach in patients with cancer (Supplementary Table 5).

A major open question is whether the residency time of the locally injected mAb within the tumour tissue is sufficient for therapeutic activity. Certain pharmaceutical formulations might help prolong the local bioavailability of such agents. For example, slow in situ release of anti-CTLA4 mAbs can be achieved through intratumoural injection as emulsions formulated with ethiodized oil and poly(lactic-co-glycolic acid) nanoparticles ${ }^{194}$. Alternatively, conjugation to an extracellular matrix super-affinity peptide derived from PlGF2 results in better tissue retention and lower blood concentrations of anti-CTLA4 and anti-PD-1 mAbs, which is associated with a reduced incidence of systemic irAEs and enhanced antitumour efficacy relative to the unconjugated mAbs in preclinical models ${ }^{195}$.

\section{Local administration of immune cells}

Human immune cells, including DCs, T cells and NK cells, can be isolated from patients or donors and subsequently expanded and manipulated by modifying the culture conditions or through genetic engineering to generate clinical grade cell therapies. For example, much clinical data has been published regarding the use of ex vivo differentiated or directly isolated DCs to formulate therapeutic cancer vaccines, although thus far the efficacy of such vaccines has been modest ${ }^{196}$. An alternative approach consists of injecting DCs intratumourally, where they are optimally positioned to encounter, process and cross-present tumour-associated antigens ${ }^{197}$. The beauty of this strategy is that the DCs might act as an in situ vaccine to orchestrate an endogenous immune response. Few clinical studies of this approach have been reported, some involving gene transfer to the DCs prior to intratumoural delivery, although numerous trials are currently ongoing (Supplementary Table 6). However, excellent results have been obtained in mouse models, particularly with DCs engineered to express IL-12 $\left(\right.$ REF. $\left.{ }^{198}\right)$. Nonetheless, consideration must be given to the fact that the DCs are delivered into malignant tissues harbouring an abundance of substances that might alter and/or disrupt their antigen-presenting functions ${ }^{199}$, and the first clinical studies of such an approach showed limited efficacy in patients with HCC or pancreatic cancer $^{198}$.

An interesting line of research involves intratumoural injection of allogeneic, monocyte-derived, pro-inflammatory DCs after in vitro incubation with poly I:C, resiquimod and IFN $\gamma^{200}$. Following promising results in mouse models, this treatment, termed ilixadencel, has been administered intratumourally to patients with RCC, HCC or gastrointestinal stromal tumours ${ }^{200-202}$. The procedure was demonstrated to be safe, with evidence of massive infiltration of T cells, which presumably mostly recognized alloantigens, into injected tumours. However, no evidence of clinical benefit has been reported to date.

The first successful applications of adoptive $\mathrm{T}$ cell therapy in patients with melanoma or cervical cancer ${ }^{3}$ involved the systemic infusion of cultures derived from ex vivo expansion of TILs with the ability to recognize autologous tumour antigens. Intratumoural TIL therapy is now under clinical testing (NCT03362619). However, excellent results have been achieved with repeated intratumoural injection of mouse TIL cultures in syngeneic models or of human TILs into autologous tumour xenografts ${ }^{203}$. Such effects were greatly enhanced when TILs were engineered to transiently express IL-12, and further enhanced with transient expression of CD137 ligand or co-injection of an agonistic anti-CD137 mAb that resulted in complete eradication of both injected and distant tumours ${ }^{203}$. Importantly, this approach leads to endogenous $\mathrm{T}$ cell cross-priming and epitope spreading through the contribution of $\mathrm{cDC} 1 \mathrm{~s}^{203}$, probably reflecting the fact that $\mathrm{T}$ cell-mediated and NK cell-mediated cytotoxicity is a form of $\mathrm{ICD}^{204}$.

The first clinical experience with intratumoural injection of cytotoxic lymphocytes was reported two decades ago ${ }^{205}$. Specifically, lymphokine-activated killer cells (a subset of NK cells with heightened cytotoxicity towards malignant cells) were administered intracranially in patients with glioma, with concomitant systemic infusion of IL-2, resulting in local responses without serious complications ${ }^{205}$. Ongoing clinical trials are testing intratumoural injections of NK cells (Supplementary Table 6).

$\mathrm{T}$ cells engineered to express CARs have revolutionized the treatment of B cell leukaemias and lymphomas ${ }^{206}$. By contrast, the utility of CAR T cell therapy for the treatment of solid malignancies remains a matter of active research ${ }^{2}$. Delivery of the CAR T cells directly into tumours might maximize the potential of this class of therapies. Clinical experience with intracranial administration of IL-13R $\alpha 2$-targeted CAR T cells in patients with glioblastoma has been reported ${ }^{207}$. Intralesional and intraventricular delivery was feasible with appropriate neurosurgical devices, and preliminary evidence of clinical activity following local administration was obtained ${ }^{208}$; however, whether this activity surpasses that associated with systemic administration remains to be demonstrated in a head-to-head comparison. Multiplex gene editing of the T cells to enhance their persistence and to overcome immunosuppressive and resistance mechanisms in vivo will probably be required to optimize the intratumoural approach ${ }^{209}$. Preliminary results of local and intratumoural delivery of CAR T cell have been presented. For example, intrapleural injection of mesothelin-specific CAR T cells produced two complete and five partial PET responses among 20 patients 
with mesothelioma (NCT02414269) ${ }^{210}$. In addition, a pan-ErbB-targeted CAR T cell product had a good safety profile in a dose-escalation trial (NCT01818323) and resulted in disease stabilization in $9(69 \%)$ of 13 patients with head and neck SCC, although no objective responses were observed ${ }^{211}$. Local delivery of carcinoembryonic antigen (CEA)-targeted CAR T cells via the hepatic artery has also been reported in a patient with pancreatic cancer liver metastasis, resulting in a complete PET response (NCT02850536) ${ }^{212}$. The field of local and intratumoural CAR therapy is in its infancy but has promise, particularly considering that repeated administration is feasible.

\section{Dual tumour and immune targeting}

Several biotechnology strategies are being used to selectively target the activity of immunotherapies towards tumour tissues. In general, these strategies exploit selective expression of certain molecules and/or characteristic biochemical or biophysical features of the TME to confine the biodistribution and/or activity of the immunotherapy agent to the malignant tissues, thereby broadening the therapeutic index (FIG. 4). Two main approaches are under development: (1) CD3-targeted bispecific antibodies, and (2) other immunomodulatory biomolecules that accumulate or become activated selectively in tumour tissues and potentially the draining lymph nodes.

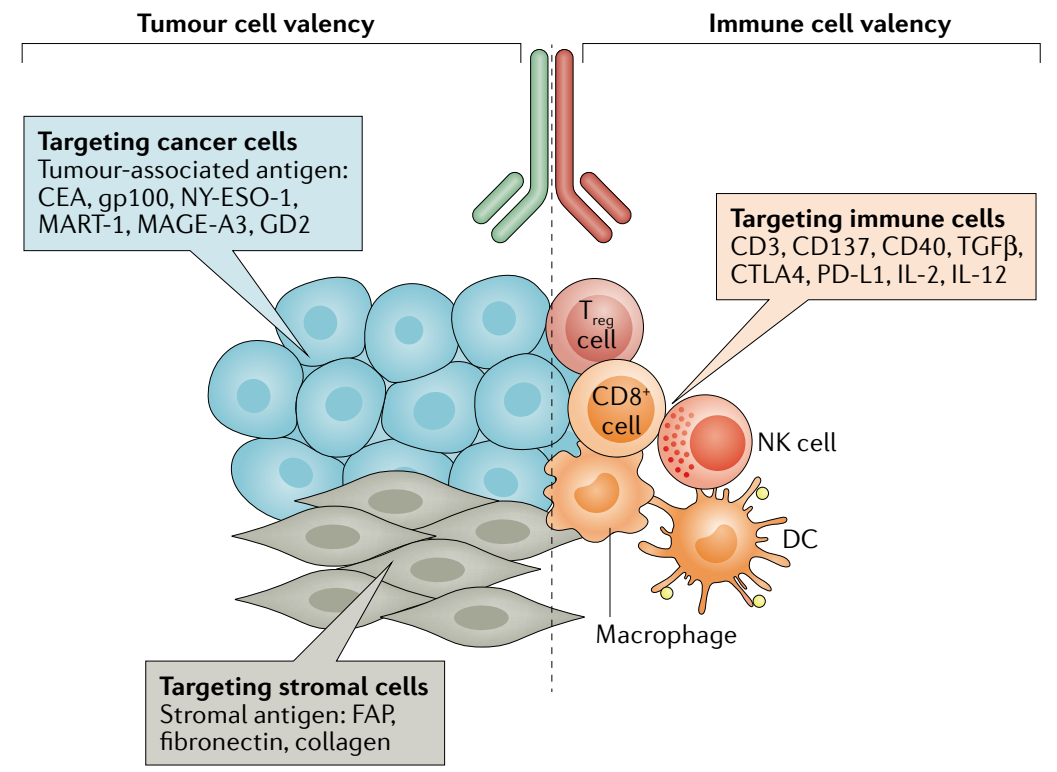

Fig. 4 | Targeted approaches to immunotherapy against solid tumours. Multiple strategies are currently being developed to promote the selective homing, accumulation and/or activation of immunotherapeutic agents inside tumours following systemic administration. These novel drugs, which include bispecific biologics, antibody Fab fragments, full antibodies, DARPins, immunocytokines and probodies, have diverse structures and target various factors present on tumour cells, in the tumour stroma and/or on immune cells to facilitate selective activation of immune responses in malignant tissues. Examples of targets for tumour cell valency or immune cell valency are listed in the figure. Such targeting strategies could also be of great interest for local immunotherapy owing to the potential to improve tumour exposure through tissuetethering and retention of the agent in the tumour microenvironment following intratumoural administration. CEA, carcinoembryonic antigen; DC, dendritic cell; FAP, fibroblast-activation protein; NK, natural killer; $\mathrm{T}_{\text {reg, }}$, regulatory $\mathrm{T}$.

\section{Bispecific T cell engagers}

Bispecific T cell engagers (BiTEs) are antibody-based agents of various formats that are able to simultaneously bind to a cell-surface tumour-associated antigen and the CD $3 \varepsilon$ component of the $\mathrm{T}$ cell receptor (TCR) to trigger $\mathrm{T}$ cell activation, thereby mimicking antigen recognition through TCR-CD3 crosslinking ${ }^{213}$. This principle underlies the efficacy of blinatumomab, an anti-CD19/CD3 BiTE approved for the treatment of B cell malignancies ${ }^{214}$. Identification of tumour-specific cell-surface antigens is a substantial challenge to the development of BiTEs targeting solid tumours. Nevertheless, several T cell engagers have been developed and tested in patients with solid tumours, including agents targeting the tumour-associated proteins EpCAM ${ }^{215-217}$ or $\mathrm{CEA}^{218}$, or MHC I-presented tumour-associated antigens derived from gp100, NY-ESO-1, MART-1 or MAGE-A3 (REF. ${ }^{219}$ ). With all these agents, systemic inflammation and cytokine-release syndromes are concerns. Therefore, $\mathrm{T}$ cell engagers are likely to be tested intratumourally in the future, exploiting their targeting properties for first-pass retention in the tumour tissue to maximize tumour cell destruction while potentially reducing the risk of toxicities. The finding that cytotoxicity induced by $\mathrm{T}$ cells and NK cells is a form of $\mathrm{ICD}^{204}$ support this strategy as an immunizing manoeuvre to elicit systemic antitumour immunity.

When considering these approaches, it becomes clear that CD3-mediated $\mathrm{T}$ cell activation (signal 1) might be insufficient for killing of epithelial cancer cells that lack co-stimulatory ligands (which induce signal 2). In fact, signal 1 alone is known to cause T cell apoptosis ${ }^{220}$. Hence, co-targeting with natural co-stimulatory ligands or $\mathrm{mAb}$ agonists of co-stimulatory receptors is of interest because of its potential to provide signal 2 for full $\mathrm{T}$ cell activation. In this regard, interesting bispecific agents have been developed that integrate anti-fibroblast activated protein (FAP) ${ }^{221}$ or anti-PD-L1 antibodies ${ }^{222}$ (for tumour targeting) with the receptor-binding regions of CD137 ligand (for co-stimulation of T cells via binding to CD137). One such agent has already entered clinical testing in combination with the anti-PD-L1 antibody atezolizumab (NCT03869190); however, combination with CD3-targeted BiTEs is perhaps a more relevant approach following the observation of potent synergy in preclinical xenograft models ${ }^{221}$.

\section{Immunocytokines and probodies}

Another strategy for local stimulation of immune cells involves genetic or chemical fusions of cytokines with antibodies targeting moieties that are enriched in tumours, with the aim of enhancing antitumour immune responses in the TME whilst minimizing systemic toxicities. The first of these 'immunocytokines' to enter clinical testing comprises IL-2 linked to a mAb targeting the ganglioside GD2, which is overexpressed on the surface of neuroblastoma cells ${ }^{223}$. This agent, hu14.18-IL2, is postulated to function through ADCC enhanced by the IL- 2 component and afucosylation ${ }^{224,225}$. In patients with neuroblastoma detectable only by ${ }^{123}$ I-metaiodobenzylguanidine scintigraphy and/or bone marrow histology, hu14.8-IL2 resulted in a CR 
rate of $21.7 \%$, whereas no responses were observed in patients with bulky radiologically detectable disease ${ }^{226}$. Randomized trials of this agent in patients with neuroblastoma are eagerly awaited, given that the naked anti-GD2 antibody dinutuximab has been approved by the FDA in combination with alternating cycles of intravenous IL-2 and GM-CSF for the treatment of patients who have responded to initial multimodal therapy ${ }^{227}$. Notably, intratumoural administration of hu14.18-IL2 is being tested in patients with advanced-stage melanoma, another tumour type in which expression of GD2 is common, including patients treated in combination with systemic immune-checkpoint inhibitors (NCT03958383; Supplementary Tables 4, 7).

Another immunocytokine is IL-2 fused to the anti-fibronectin antibody L19, which recognizes an alternatively spliced form of this extracellular matrix protein that is abundant in tumour tissues. This agent is safe when given to patients intravenously and resulted in stable disease in 17 (51\%) of 33 patients, including 15 (83\%) of 18 with RCC, although no objective responses have been reported to date ${ }^{228}$. L19-IL-2 has been administered intratumourally in combination with an analogous immunocytokine comprising L19 and TNF (L19-TNF) to 20 efficacy-evaluable patients with advanced-stage melanoma, and resulted in complete resolution of 32 tumours, including some non-injected lesions ${ }^{229}$.

A serious caveat of IL-2 relates to its role as a prominent growth and stimulating factor for CD25-expressing $\mathrm{T}_{\text {reg }}$ cells. In addition, CD25 expression on lung vascular cells is involved in toxicities of high-dose IL-2, such as vascular leak syndrome. To circumvent these problems, mutated forms of IL-2 that can bind to and signal via the IL- $2 \mathrm{R} \beta \gamma$ complex but have a much lower affinity for CD25 have been generated ${ }^{230}$. Clinical trials of immunocytokines targeting such variants of IL-2 to CEA (NCT02004106 and NCT02350673) ${ }^{231}$ or FAP (NCT02627274, NCT03875079, NCT03063762, NCT03386721 and NCT03193190) $)^{232}$ are underway. A disadvantage of these approaches is that the variant forms of IL-2 result in rapid systemic expansion of $\mathrm{T}$ cells and NK cells, resulting in pro-inflammatory adverse effects that are likely to be dependent mostly on the peak concentration of the drug. Thus, subcutaneous, intratumoural and protracted infusions might be needed for optimal results.

TGF $\beta$ is a crucial cytokine involved in promoting epithelial-mesenchymal transition and the induction of immunosuppressive $\mathrm{T}_{\text {reg }}$ cells, which support tumour progression ${ }^{233}$. In situ targeting of TGF $\beta$ has been made possible with the development of bintrafusp alfa, a fusion of a tumour-targeted anti-PD-L1 antibody (avelumab) with a TGF $\beta$-receptor trap. Notably, this agent produced an ORR of $39 \%$ in patients with HPV-related carcinoma $^{234}$.

A novel approach that is being tested in clinical trials involves tumour-tissue targeting of IL-12. As discussed, this cytokine is among the most potent immunotherapies, but its use is curtailed by systemic DLTs. NHSIL12 is an immunocytokine comprising two single-chain IL-12 heterodimers fused to an antibody targeting histones bound to cell-free dsDNA, which is abundant in necrotic tumours ${ }^{235}$ as well as tumour-associated neutrophil extracellular traps ${ }^{236}$. This immunocytokine increases $\mathrm{T}$ cell diversity and density in human tumours, although no objective clinical responses have been observed to date ${ }^{237}$. NHS-IL12 is currently under clinical investigation in combination with bintrafusp alfa across various malignancies (NCT04708470, NCT04235777, NCT04756505 and NCT04633252). Again, the IL-12 component of NHS-IL12 is active systemically, which raises safety concerns; therefore, testing of intratumoural delivery is warranted, particularly considering the excellent results obtained with pembrolizumab combined with local electroporation of tumours with IL-12-encoding plasmid DNA ${ }^{156}$.

Biotechnology can also provide the means for selective activation of an immunotherapy prodrug within the TME. Several features of the malignant tissue can be exploited for this purpose, such as the activity of metalloproteinases, low $\mathrm{pH}$, an abundance of extracellular ATP or hypoxia. The most advanced strategy of this kind in clinical development is based on masking of the antigen-binding site of mAbs with peptides tethered to the light chains via a cleavable polypeptide linker ${ }^{238}$; thus, proteases enriched and active in the TME can 'unmask' the antigen-binding activity of the mAb. This 'probody' approach is being used for functional tumour-targeting of mAbs that might otherwise have serious safety concerns as a result of their systemic biodistribution ${ }^{238}$. In the context of cancer immunotherapy, this concept is particularly attractive for anti-CTLA4 mAbs, agonist anti-CD137 mAbs and TGF $\beta$ antagonists, among others. The probody strategy could potentially be extended to other agents, such as cytokines (pro-cytokines). Anti-CTLA4 and anti-PD-L1 probodies are already being tested in FIH trials, with results eagerly awaited, and might be especially suitable for neoadjuvant and/or adjuvant therapy considering that a more favourable safety profile is particularly important for patients who are surgically treated with curative intent.

In a very innovative approach, an agonistic $\mathrm{mAb}$ targeting human CD137 has been selected in such a fashion that it only binds to its target in tissues with a high concentration of ATP, as is usually found in tumours. This mAb, STA551, was active against tumours in human CD137 knock-in mice while avoiding severe liver inflammation-related toxicities observed with other anti-CD137 mAbs, thus suggesting the potential for clinical translation of this immunotherapy ${ }^{239}$.

\section{Conclusions}

Direct intratumoural administration offers an opportunity to maximize the therapeutic index of several immunomodulatory therapies by reducing systemic exposure $^{240}$. In principle, the aim of such in situ immune stimulation is to use the tumour as its own vaccine and either prime or enhance a pre-existing antitumour immune response ${ }^{10}$. However, the clinical, radiological and biological data collected in most initial trials of intratumoural immunotherapies do not precisely distinguish between local and distant responses; 


\section{Box 3 | Logistics and practical considerations for clinical development}

Novel intratumoural and tumour-targeted approaches to immunotherapy are challenging the classic clinical development paradigms of oncology drugs. Most of these immunotherapies do not meet the standard dose-limiting toxicity (DLT) criteria: often, the treatment-related adverse events occur beyond the classic 28-day DLT period and are not dose-dependent, but rather patient-dependent. Moreover, the aim of dose escalation within a first-in-human $(\mathrm{FIH})$ trial of such strategies is no longer to reach a maximum tolerated dose but rather an optimal biological dose (OBD). The definition of the OBD is dependent on pharmacodynamic biomarkers related to target engagement. Most of the pharmacodynamic changes with intratumoural or tumour-targeted treatments occur in the tumour bed, which therefore mandates pretreatment and on-treatment tumour biopsy sampling in order to determine the OBD. Taking into account the need to acquire robust pharmacodynamic data with replicate and the inter-individual variability, the historical $3+3$ clinical trial design for a FIH trial is not sufficient to determine the OBD and thus the ideal dosage. Specific clinical trial designs and FIH phase I trials with larger cohorts are therefore needed to develop these novel therapeutic strategies ${ }^{261}$. Furthermore, conventional radiological assessment according to the Response Criteria in Solid Tumors version 1.1 (RECIST1.1) is not suited to intratumoural therapies and does not separately capture the responses of injected versus non-injected tumours while also considering atypical tumour responses (for example, pseudoprogression, transient new lesions and delayed responses). To foster the development of intratumoural immunotherapies, new radiological Response Criteria for Intratumoral Immunotherapy in Solid Tumors (itRECIST) have been proposed ${ }^{262}$. However, the correlations between responses as per these criteria and overall survival remain to be determined. Last but not least, the clinical development of intratumoural immunotherapies requires dedicated multidisciplinary staff to prescribe, execute and monitor the therapeutic procedures in accordance with the clinical trial protocol ${ }^{263}$.

deciphering these effects in injected and non-injected tumours will thus be a crucial objective for future trials. PD-1 or PD-L1 inhibitors might be optimally effective when delivered systemically, although the therapeutic value of local administration of these agents remains largely unknown. Nonetheless, preclinical and clinical evidence indicates that PRR agonists and oncolytic viruses are more active upon local delivery and might synergize with systemic immune-checkpoint inhibitors.
Such strategies are currently being tested in phase III registration trials (NCT03445533 and NCT02263508).

Intratumoural immunotherapies can be administered by either direct or image-guided injections; therefore, the efficacy of local immunotherapy might be operator-dependent, relying on the quality of in situ delivery. This technical aspect is a serious concern for the large-scale development of intratumoural therapies, especially when contemplating multicentre phase III registration trials. Beyond the need for harmonization of technical practice, clinical trial designs and end points, a need also exists for close monitoring to capture suboptimal or failed delivery of intratumoural immunotherapy in order to adjust the interpretation of the clinical outcomes.

Novel localized immunotherapy strategies and agents are revolutionizing our understanding and treatment of cancers, and will probably also transform the way we perform drug development in oncology. Nevertheless, these approaches pose complex logistical challenges (BOX 3) and require a change in the paradigms of oncology practice.

The efficacy of such localized immunotherapy is expected to be higher in patients with early stage cancers, in accordance with the historical data on T-VEC (which are associated with improved OS in those with stage IIIB-M1a melanoma but not in those with M1b/c disease $)^{96}$. Therefore, these intratumoural and tumour tissue-targeted immunotherapies will probably be of specific value for localized cancers and should, consequently, dramatically change the outcomes and the toxicity profile of immunotherapies, in particular, when deployed for local neoadjuvant therapy in patients with locally advanced and resectable cancers ${ }^{241}$.

Published online 18 May 2021
1. Ribas, A. \& Wolchok, J. D. Cancer immunotherapy using checkpoint blockade. Science 359, 1350-1355 (2018).

2. Guedan, S., Ruella, M. \& June, C. H. Emerging cellular therapies for cancer. Annu. Rev. Immunol. 37 , 145-171 (2019).

3. Rosenberg, S. A. \& Restifo, N. P. Adoptive cell transfer as personalized immunotherapy for human cancer. Science 348, 62-68 (2015).

4. Cuesta, A. M., Sainz-Pastor, N., Bonet, J., Oliva, B. \& Alvarez-Vallina, L. Multivalent antibodies: when design surpasses evolution. Trends Biotechnol. 28, 355-362 (2010)

5. Melero, I., Rouzaut, A., Motz, G. T. \& Coukos, G. T-cell and NK-cell infiltration into solid tumors: a key limiting factor for efficacious cancer immunotherapy. Cancer Discov, 4, 522-526 (2014).

6. Postow, M. A., Sidlow, R. \& Hellmann, M. D. Immune-related adverse events associated with immune checkpoint blockade. N. Engl. J. Med. 378, 158-168 (2018)

7. Leonard, J. P. et al. Effects of single-dose interleukin-12 exposure on interleukin-12-associated toxicity and interferon-gamma production. Blood $\mathbf{9 0}$, 2541-2548 (1997).

8. Ascierto, P. A. et al. Overall survival at 5 years of follow-up in a phase III trial comparing ipilimumab $10 \mathrm{mg} / \mathrm{kg}$ with $3 \mathrm{mg} / \mathrm{kg}$ in patients with advanced melanoma. J. Immunother. Cancer 8, e000391 (2020).

9. Wang, Y. L., Peng, H. H., Su, S. Y. \& Lin, C. T. Combined immunotherapy (OK-432, IL-2) with chemotherapy decrease the recurrence rate in advanced ovarian cancer. Reprod. Sci. 26, 244-249 (2019).

10. Marabelle, A., Tselikas, L., de Baere, T. \& Houot, R Intratumoral immunotherapy: using the tumor as the remedy. Ann. Oncol. 28, xii33-xii43 (2017).
11. Margolin, K. et al. Phase I trial of ALT-803, a novel recombinant IL15 complex, in patients with advanced solid tumors. Clin. Cancer Res. 24, 5552-5561 (2018).

12. Barlesi, F. et al. Phase Ib study of selicrelumab (CD40 agonist) in combination with atezolizumab (anti-PD-L1) in patients with advanced solid tumors [abstract 291]. J. Immunother. Cancer 8 (Suppl. 3), A178 (2020)

13. Chandrasekaran, S. \& King, M. R. Microenvironment of tumor-draining lymph nodes: opportunities for liposome-based targeted therapy. Int. J. Mol. Sci. 15 20209-20239 (2014).

14. Petitprez, F. et al. B cells are associated with survival and immunotherapy response in sarcoma. Nature 577, 556-560 (2020).

15. Helmink, B. A. et al. B cells and tertiary lymphoid structures promote immunotherapy response. Nature 577, 549-555 (2020).

16. Vormehr, M., Tureci, O. \& Sahin, U. Harnessing tumor mutations for truly individualized cancer vaccines. Annu. Rev. Med. 70, 395-407 (2019).

17. Linette, G. P. et al. Immunological ignorance is an enabling feature of the oligo-clonal T cell response to melanoma neoantigens. Proc. Natl Acad. Sci. USA 116, 23662-23670 (2019).

18. Harrington, K., Freeman, D. J., Kelly, B., Harper, J \& Soria, J. C. Optimizing oncolytic virotherapy in cancer treatment. Nat. Rev. Drug Discov. 18, 689-706 (2019).

19. Coley, W. B. The treatment of malignant tumors by repeated inoculations of erysipelas. With a report of ten original cases. Clin. Orthop. Relat. Res. 262, 3-11 (1991).

20. Nauts, H. C., Swift, W. E. \& Coley, B. L. The treatment of malignant tumors by bacterial toxins as developed by the late William B. Coley, M.D., reviewed in the light of modern research. Cancer Res. 6, 205-216 (1946).

21. Tsung, K. \& Norton, J. A. Lessons from Coley's toxin. Surg. Oncol. 15, 25-28 (2006).

22. Hoption Cann, S. A., van Netten, J. P. \& van Netten, C. Dr William Coley and tumour regression: a place in history or in the future. Postgrad. Med. J. 79 672-680 (2003).

23. Morales, A., Eidinger, D. \& Bruce, A. W. Intracavitary Bacillus Calmette-Guerin in the treatment of superficial bladder tumors. J. Urol. 116, 180-183 (1976).

24. Mori, K., Lamm, D. L. \& Crawford, E. D. A trial of bacillus Calmette-Guérin versus adriamycin in superficial bladder cancer: a South-West Oncology Group Study. Urol. Int. 41, 254-259 (1986).

25. Mostafid, A. H., Palou Redorta, J., Sylvester, R. \& Witjes, J. A. Therapeutic options in high-risk nonmuscle-invasive bladder cancer during the current worldwide shortage of bacille Calmette-Guérin. Eur. Urol. 67, 359-360 (2015).

26. van Puffelen, J. H. et al. Trained immunity as a molecular mechanism for BCG immunotherapy in bladder cancer. Nat. Rev. Urol. 17, 513-525 (2020).

27. Yang, J. et al. Insights into local tumor microenvironment immune factors associated with regression of cutaneous melanoma metastases by Mycobacterium bovis bacille Calmette-Guérin. Front. Oncol. 7, 61 (2017).

28. Svejda, J., Mechl, Z., Sopkova, B. \& Foukal, T Histologic changes in the human skin melanoma after intratumorous treatment with BCG. Neoplasma 26, 215-221 (1979).

29. Agarwala, S. S., Neuberg, D., Park, Y. \& Kirkwood, J. M Mature results of a phase III randomized trial of bacillus Calmette-Guerin (BCG) versus observation and 
BCG plus dacarbazine versus BCG in the adjuvant therapy of American Joint Committee on Cancer Stage I-III melanoma (E1673): a trial of the Eastern Oncology Group. Cancer 100, 1692-1698 (2004).

30. Fisher, R. I. et al. Adjuvant immunotherapy or chemotherapy for malignant melanoma. Preliminary report of the National Cancer Institute randomized clinical trial. Surg. Clin. North Am. 61, 1267-1277 (1981).

31. Chen, D. S. \& Mellman, I. Oncology meets immunology: the cancer-immunity cycle. Immunity 39 , $1-10(2013)$

32. Iwasaki, A. \& Medzhitov, R. Control of adaptive immunity by the innate immune system. Nat. Immunol. 16, 343-353 (2015).

33. Matzinger, P. An innate sense of danger. Ann. NY Acad. Sci. 961, 341-342 (2002)

34. Hemmi, H. et al. A Toll-like receptor recognizes bacterial DNA. Nature 408, 740-745 (2000).

35. Kandimalla, E. R. et al. Immunomodulatory oligonucleotides containing a cytosine-phosphate-2 deoxy-7-deazaguanosine motif as potent toll-like receptor 9 agonists. Proc. Natl Acad. Sci. USA 102, 6925-6930 (2005)

36. Wooldridge, J. E. \& Weiner, G. J. CpG DNA and cancer immunotherapy: orchestrating the antitumor immune response. Curr. Opin. Oncol. 15, 440-445 (2003).

37. Krieg, A. M. Development of TLR9 agonists for cancer therapy. J. Clin. Invest. 117, 1184-1194 (2007).

38. Manegold, C. et al. Randomized phase II trial of a toll-like receptor 9 agonist oligodeoxynucleotide, PF-3512676, in combination with first-line taxane plus platinum chemotherapy for advanced-stage nonsmall-cell lung cancer. J. Clin. Oncol. 26, 3979-3986 (2008).

39. Hirsh, V. et al. Randomized phase III trial of paclitaxel/ carboplatin with or without PF-3512676 (Toll-like receptor 9 agonist) as first-line treatment for advanced non-small-cell lung cancer. J. Clin. Oncol. 29, 2667-2674 (2011)

40. Belani, C. P. et al. Phase 2 trial of erlotinib with or without PF-3512676 (CPG 7909, a Toll-like receptor 9 agonist) in patients with advanced recurrent EGFRpositive non-small cell lung cancer. Cancer Biol. Ther. 14, 557-563 (2013)

41. Brody, J. D. et al. In situ vaccination with a TLR9 agonist induces systemic lymphoma regression: a phase I/II study. J. Clin. Oncol. 28, 4324-4332 (2010).

42. Kim, Y. H. et al. In situ vaccination against mycosis fungoides by intratumoral injection of a TLR9 agonist combined with radiation: a phase $1 / 2$ study. Blood 119, 355-363 (2012)

43. Frank, M. J. et al. In situ vaccination with a TLR9 agonist and local low-dose radiation induces systemic responses in untreated indolent lymphoma. Cancer Discov. 8, 1258-1269 (2018)

44. Ribas, A. et al. SD-101 in combination with pembrolizumab in advanced melanoma: results of a phase Ib, multicenter study. Cancer Discov. 8 1250-1257 (2018)

45. Li, J. et al. Lymphoma immunotherapy with $\mathrm{CpC}$ oligodeoxynucleotides requires TLR9 either in the host or in the tumor itself. J. Immunol. 179, 2493-2500 (2007).

46. Haymaker, C. et al. Final results from ILLUMINATE-204, a phase I/II trial of intratumoral tilsotolimod in combination with ipilimumab in PD- 1 inhibitor refractory advanced melanoma [abstract 1083MO]. Ann. Oncol. 31 (Suppl. 4), S736 (2020).

47. Poltorak, A. et al. Defective LPS signaling in $\mathrm{C} 3 \mathrm{H} / \mathrm{HeJ}$ and C57BL/10ScCr mice: mutations in Tlr4 gene. Science 282, 2085-2088 (1998).

48. Weighardt, H. et al. Identification of a TLR4- and TRIF-dependent activation program of dendritic cells. Eur. J. Immunol. 34, 558-564 (2004).

49. Apetoh, L. et al. Toll-like receptor 4-dependent contribution of the immune system to anticancer chemotherapy and radiotherapy. Nat. Med. 13 1050-1059 (2007)

50. Persing, D. H. et al. Taking toll: lipid A mimetics as adjuvants and immunomodulators. Trends Microbiol. 10, S32-S37 (2002).

51. Chicoine, M. R., Won, E. K. \& Zahner, M. C. Intratumoral injection of lipopolysaccharide causes regression of subcutaneously implanted mouse glioblastoma multiforme. Neurosurgery 48, 607-614; discussion 614-5 (2001).

52. Bhatia, S. et al. Intratumoral G100, a TLR4 agonist, induces antitumor immune responses and tumor regression in patients with Merkel cell carcinoma. Clin. Cancer Res. 25, 1185-1195 (2019).
53. Flowers, C. et al. Intratumoral G100 induces systemic immunity and abscopal tumor regression in patients with follicular lymphoma: results of a phase $1 / 2$ study examining $\mathrm{G} 100$ alone and in combination with pembrolizumab [abstract]. Blood 130 (Suppl. 1), 2771 (2017).

54. Flowers, C. R. et al. Long term follow-up of a phase 2 study examining intratumoral G100 alone and in combination with pembrolizumab in patients with follicular lymphoma [abstract]. Blood 132 (Suppl. 1), 2892 (2018)

55. Alexopoulou, L., Holt, A. C., Medzhitov, R. \& Flavell, R. A. Recognition of double-stranded RNA and activation of NF-кB by Toll-like receptor 3. Nature 413, 732-738 (2001).

56. Nemes, M. M., Tytell, A. A., Lampson, G. P., Field, A. K \& Hilleman, M. R. Inducers of interferon and host resistance. VI. Antiviral efficacy of poly I:C in animal models. Proc. Soc. Exp. Biol. Med. 132, 776-783 (1969).

57. Schulz, O et al. Toll-like receptor 3 promotes crosspriming to virus-infected cells. Nature $433,887-892$ (2005).

58. Degre, M. \& Elgjo, K. Methylcholanthrene-induced skin carcinogenesis modified by treatment with polyinosinic:polycytidylic acid (poly I:C). Acta Pathol. Microbiol. Scand. A 79, 687-688 (1971).

59. Fujimura, T., Nakagawa, S., Ohtani, T., Ito, Y. $\delta$ Aiba, S. Inhibitory effect of the polyinosinicpolycytidylic acid/cationic liposome on the progression of murine B16F10 melanoma. Eur. J. Immunol. 36, 3371-3380 (2006).

60. Salazar, A. M., Erlich, R. B., Mark, A., Bhardwaj, N. \& Herberman, R. B. Therapeutic in situ autovaccination against solid cancers with intratumoral poly-ICLC: case report, hypothesis, and clinical trial. Cancer Immunol. Res. 2, 720-724 (2014).

61. Rodriguez-Ruiz, M. E. et al. Combined immunotherap encompassing intratumoral poly-ICLC, dendritic-cell vaccination and radiotherapy in advanced cancer patients. Ann. Oncol. 29, 1312-1319 (2018).

62. Caskey, $M$ et al. Synthetic double-stranded RNA induces innate immune responses similar to a live viral vaccine in humans. J. Exp. Med. 208, 2357-2366 (2011).

63. Okada, H. et al. Induction of CD8 $8^{+} \mathrm{T}$-cell responses against novel glioma-associated antigen peptides and clinical activity by vaccinations with $\alpha$-type 1 polarized dendritic cells and polyinosinic-polycytidylic acid stabilized by lysine and carboxymethylcellulose in patients with recurrent malignant glioma. J. Clin. Oncol. 29, 330-336 (2011).

64. Aznar, M. A. et al. Immunotherapeutic effects of intratumoral nanoplexed poly I:C. J. Immunother. Cancer 7, 116 (2019)

65. Tormo, D. et al. Targeted activation of innate immunity for therapeutic induction of autophagy and apoptosis in melanoma cells. Cancer Cell 16, 103-114 (2009).

66. Kalbasi, A. et al. Uncoupling interferon signaling and antigen presentation to overcome immunotherapy resistance due to JAK1 loss in melanoma. Sci. Transl Med. 12, eabb0152 (2020).

67. Marquez-Rodas, I. et al. Intratumoral nanoplexed poly I:C BO-112 in combination with systemic anti-PD-1 for patients with anti-PD-1-refractory tumors. Sci. Trans/ Med. 12, eabb0391 (2020)

68. Barral, P. M. et al. Functions of the cytoplasmic RNA sensors RIG-I and MDA-5: key regulators of innate immunity. Pharmacol. Ther. 124, 219-234 (2009).

69. Diebold, S. S. et al. Viral infection switches nonplasmacytoid dendritic cells into high interferon producers. Nature 424, 324-328 (2003).

70. Ishizuka, J. J. et al. Loss of ADAR1 in tumours overcomes resistance to immune checkpoint blockade. Nature 565, 43-48 (2019).

71. Liu, H. et al. Tumor-derived IFN triggers chronic pathway agonism and sensitivity to ADAR loss. Nat. Med. 25, 95-102 (2019).

72. Diebold, S. S., Kaisho, T., Hemmi, H., Akira, S. \& Reis e Sousa, C. Innate antiviral responses by means of TLR7-mediated recognition of single-stranded RNA Science 303, 1529-1531 (2004).

73. Kariko, K., Buckstein, M., Ni, H. \& Weissman, D. Suppression of RNA recognition by Toll-like receptors: the impact of nucleoside modification and the evolutionary origin of RNA. Immunity 23, 165-175 (2005).

74. Hemmi, H. et al. Small anti-viral compounds activate immune cells via the TLR7 MyD88-dependent signaling pathway. Nat. Immunol. 3, 196-200 (2002).
75. Sidky, Y. A. et al. Inhibition of murine tumor growth by an interferon-inducing imidazoquinolinamine Cancer Res. 52, 3528-3533 (1992).

76. Syed, T. A., Ahmadpour, O. A., Ahmad, S. A. \& Ahmad, S. H. Management of female genital warts with an analog of imiquimod $2 \%$ in cream: a randomized, double-blind, placebo-controlled study. J. Dermatol. 25, 429-433 (1998).

77. Stary, G. et al. Tumoricidal activity of TLR7/8-activated inflammatory dendritic cells. J. Exp. Med. 204 1441-1451 (2007).

78. van Seters, M. et al. Treatment of vulvar intraepithelial neoplasia with topical imiquimod. N. Engl. J. Med. 358, 1465-1473 (2008)

79. Adams, S. et al. Topical TLR7 agonist imiquimod can induce immune-mediated rejection of skin metastases in patients with breast cancer. Clin. Cancer Res. 18 , 6748-6757 (2012).

80. Rook, A. H. et al. Topical resiquimod can induce disease regression and enhance T-cell effector functions in cutaneous T-cell lymphoma. Blood 126, 1452-1461 (2015).

81. Chen, Q., Sun, L. \& Chen, Z. J. Regulation and function of the CGAS-STING pathway of cytosolic DNA sensing. Nat. Immunol. 17, 1142-1149 (2016).

82. Yang, Y. G., Lindahl, T. \& Barnes, D. E. Trex 1 exonuclease degrades SSDNA to prevent chronic checkpoint activation and autoimmune disease. Cell 131, 873-886 (2007)

83. Fuertes, M. B. et al. Host type I IFN signals are required for antitumor $\mathrm{CD}^{+} \mathrm{T}$ cell responses through CD8 $\alpha^{+}$dendritic cells. J. Exp. Med. 208, 2005-2016 (2011).

84. Woo, S. R. et al. STING-dependent cytosolic DNA sensing mediates innate immune recognition of immunogenic tumors. Immunity 41, 830-842 (2014).

85. Vanpouille-Box, C. et al. DNA exonuclease Trex 1 regulates radiotherapy-induced tumour immunogenicity. Nat. Commun. 8, 15618 (2017)

86. Corrales, L. et al. Direct activation of STING in the tumor microenvironment leads to potent and systemic tumor regression and immunity. Cell Rep. 11 1018-1030 (2015).

87. Lara, P. N. Jr. et al. Randomized phase III placebocontrolled trial of carboplatin and paclitaxel with or without the vascular disrupting agent vadimezan (ASA404) in advanced non-small-cell lung cancer. J. Clin. Oncol. 29, 2965-2971 (2011).

88. Harrington, K. J. et al. Preliminary results of the first-in-human (FIH) study of MK-1454, an agonist of stimulator of interferon genes (STING), as monotherapy or in combination with pembrolizumab (pembro) in patients with advanced solid tumors or lymphomas [abstract LBA 15]. Ann. Oncol. 29 (Suppl. 8), viii712 (2018).

89. Meric-Bernstam, F. et al. Phase lb study of MIW815 (ADU-S100) in combination with spartalizumab (PDR001) in patients (pts) with advanced/metastatic solid tumors or lymphomas [abstract]. J. Clin. Oncol. 37 (Suppl. 15), 2507 (2019).

90. Flood, B. A., Higgs, E. F., Li, S., Luke, J. J. \& Gajewski, T. F. STING pathway agonism as a cancer therapeutic. Immunol. Rev. 290, 24-38 (2019).

91. Giguere, C. M. et al. Treatment of lymphangiomas with OK-432 (Picibanil) sclerotherapy: a prospective multiinstitutional trial. Arch. Otolaryngol. Head. Neck Surg. 128, 1137-1144 (2002)

92. Aluffi Valletti, P. et al. A single-center experience in the management of head and neck lymphangiomas. Oral. Maxillofac. Surg. 24, 109-115 (2020).

93. Kasahara, K. et al. Randomized phase II trial of OK-432 in patients with malignant pleural effusion due to non-small cell lung cancer. Anticancer Res. 26 1495-1499 (2006)

94. Yamaguchi, Y. et al. Locoregional immunotherapy of malignant effusion from colorectal cancer using the streptococcal preparation OK-432 plus interleukin-2: induction of autologous tumor-reactive $\mathrm{CD}^{+} \mathrm{Th} 1 \mathrm{killer}$ lymphocytes. Br. J. Cancer 89, 1876-1884 (2003).

95. Hirayama, M. et al. Overcoming regulatory T-cell suppression by a lyophilized preparation of Streptococcus pyogenes. Eur. J. Immunol. 43, 989-1000 (2013).

96. Andtbacka, R. H. I. et al. Final analyses of OPTiM: a randomized phase III trial of talimogene laherparepvec versus granulocyte-macrophage colony-stimulating factor in unresectable stage III-IV melanoma. J. Immunother. Cancer 7, 145 (2019).

97. Andtbacka, R. H. et al. Talimogene laherparepvec improves durable response rate in patients with advanced melanoma. J. Clin. Oncol. 33, 2780-2788 (2015). 
98. Hamid, O. et al. Five-year survival outcomes for patients with advanced melanoma treated with pembrolizumab in KEYNOTE-001. Ann. Oncol. 30 582-588 (2019)

99. Andtbacka, R. H. et al. Patterns of clinical response with talimogene laherparepvec (T-VEC) in patients with melanoma treated in the OPTiM phase III clinical trial. Ann. Surg. Oncol. 23, 4169-4177 (2016).

100. Dillman, R. O. An update on GM-CSF and its potential role in melanoma management. Melanoma Manag. 7 MMT49 (2020).

101. Puzanov, I. et al. Talimogene laherparepvec in combination with ipilimumab in previously untreated, unresectable stage IIIB-IV melanoma. J. Clin. Oncol. 34, 2619-2626 (2016)

102. Ribas, A. et al. Oncolytic virotherapy promotes intratumoral T cell infiltration and improves anti-PD-1 immunotherapy. Cell 170, 1109-1119.e10 (2017).

103. Middleton, M. et al. An open-label, multicenter, phase $1 / 2$ clinical trial of Rp 1, an enhanced potency oncolytic Hsv, combined with nivolumab: updated results from the skin cancer cohorts [abstract 422]. J. Immunother Cancer 8 (Suppl. 3), A257 (2020)

104. Heo, J. et al. Randomized dose-finding clinical trial of oncolytic immunotherapeutic vaccinia JX-594 in liver cancer. Nat. Med. 19, 329-336 (2013).

105. Kim, M. K. et al. Oncolytic and immunotherapeutic vaccinia induces antibody-mediated complementdependent cancer cell lysis in humans. Sci. Transl Med. 5, 185ra63 (2013)

106. Transgene. Transgene provides an update after the interim futility analysis of the PHOCUS study of PexaVec in liver cancer. Transgene https://www.transgene.fr en/news/\#pressreleases (2019).

107. Zamarin, D. et al. Localized oncolytic virotherapy overcomes systemic tumor resistance to immune checkpoint blockade immunotherapy. Sci. Trans/ Med. 6, 226ra32 (2014)

108. Curti, B. D. et al. Activity of a novel immunotherapy combination of intralesional Coxsackievirus A21 and systemic ipilimumab in advanced melanoma patients previously treated with anti-PD1 blockade therapy [abstract]. J. Clin. Oncol. 35 (Suppl. 15), 3014 (2017)

109. Lang, F. F. et al. Phase I study of DNX-2401 (Delta-24-RGD) oncolytic adenovirus: replication and immunotherapeutic effects in recurrent malignant glioma. J. Clin. Oncol. 36, 1419-1427 (2018).

110. Rosa, K. Japanese approval sought for oncolytic virus teserpaturev for malignanat glioma. OncLive https:// www.onclive.com/view/japanese-approval-soughtfor-oncolytic-virus-teserpaturev-for-malignant-glioma (2021).

111. Nakao, S. et al. Intratumoral expression of IL-7 and IL-12 using an oncolytic virus increases systemic sensitivity to immune checkpoint blockade. Sci. Trans/ Med. 12, eaax7992 (2020).

112. Kepp, O., Marabelle, A., Zitvogel, L. \& Kroemer, G. Oncolysis without viruses - inducing systemic anticancer immune responses with local therapies. Nat. Rev. Clin. Oncol. 17, 49-64 (2020).

113. Swift, L., Zhang, C., Trippett, T. \& Narendran, A Potent in vitro and xenograft antitumor activity of a novel agent, PV-10, against relapsed and refractory neuroblastoma. Onco Targets Ther. 12, 1293-1307 (2019).

114. Toomey, P. et al. Intralesional injection of rose bengal induces a systemic tumor-specific immune response in murine models of melanoma and breast cancer. PLOS ONE 8, e68561 (2013).

115. Read, T. A. et al. Intralesional PV-10 for the treatment of in-transit melanoma metastases - Results of a prospective, non-randomized, single center study. J. Surg. Oncol. 117, 579-587 (2018).

116. Foote, M. et al. Results of a phase II, open-label, non-comparative study of intralesional PV-10 followed by radiotherapy for the treatment of in-transit or metastatic melanoma. J. Surg. Oncol. 115, 891-897 (2017).

117. Liu, H. et al. T cell mediated immunity after combination therapy with intralesional PV-10 and blockade of the PD-1/PD-L1 pathway in a murine melanoma model. PLOS ONE 13, e0196033 (2018).

118. De Ridder, T. R. et al. Randomized controlled clinical study evaluating the efficacy and safety of intratumoral treatment of canine mast cell tumors with tigilanol tiglate (EBC-46). J. Vet. Intern. Med. 35 415-429 (2020)

119. Panizza, B. J. et al. Phase I dose-escalation study to determine the safety, tolerability, preliminary efficacy and pharmacokinetics of an intratumoral injection of tigilanol tiglate (EBC-46). EBioMedicine 50, 433-441 (2019).
120. Boyle, G. M. et al. Intra-lesional injection of the novel PKC activator EBC- 46 rapidly ablates tumors in mouse models. PLOS ONE 9, e 108887 (2014).

121. Zanin-Zhorov, A., Dustin, M. L. \& Blazar, B. R. PKC-theta function at the immunological synapse: prospects for therapeutic targeting. Trends Immunol. 32, 358-363 (2011)

122. Anel, A. et al. Protein kinase C- $\theta$ (PKC- $\theta$ ) in natural killer cell function and anti-tumor immunity. Front Immunol. 3, 187 (2012).

123. Zanin-Zhorov, A. et al. Protein kinase $\mathrm{C}-\theta$ mediates negative feedback on regulatory $T$ cell function. Science 328, 372-376 (2010)

124. Kong, K. F. et al. Protein kinase C- $\eta$ controls CTLA-4 mediated regulatory T cell function. Nat. Immunol. 15 , 465-472 (2014)

125. Tornesello, A. L, Borrelli, A., Buonaguro, L, Buonaguro, F. M. \& Tornesello, M. L. Antimicrobial peptides as anticancer agents: functional properties and biological activities. Molecules 25, 2850 (2020).

126. Zhou, H. et al. The oncolytic peptide LTX-315 triggers immunogenic cell death. Cell Death Dis. 7, e2134 (2016).

127. Zhou, H. et al. The oncolytic peptide LTX-315 kills cancer cells through Bax/Bak-regulated mitochondrial membrane permeabilization. Oncotarget 6 , 26599-26614 (2015).

128. Baurain, J. F. et al. A phase I study of the oncolytic peptide LTX-315 generates de novo T-cell responses and clinical benefit in patients with advanced sarcoma [abstract]. Cancer Res. 79 (Suppl. 13), CT010 (2019).

129. Dubrot, J. et al. Intratumoral injection of interferon- $\alpha$ and systemic delivery of agonist anti-CD 137 monoclonal antibodies synergize for immunotherapy. Int. J. Cancer 128, 105-118 (2011).

130. Vom Berg, J. et al. Intratumoral IL-12 combined with CTLA-4 blockade elicits T cell-mediated glioma rejection. J. Exp. Med. 210, 2803-2811 (2013).

131. Negrier, S. et al. Recombinant human interleukin-2, recombinant human interferon alfa-2a, or both in metastatic renal-cell carcinoma. N. Engl. J. Med. 338 1272-1278 (1998).

132. Berraondo, P. et al. Cytokines in clinical cancer immunotherapy. Br. J. Cancer 120, 6-15 (2019).

133. Wrangle, J. M. et al. ALT-803, an IL-15 superagonist, in combination with nivolumab in patients with metastatic non-small cell lung cancer: a nonrandomised, open-label, phase $1 \mathrm{~b}$ trial. Lancet Oncol. 19, 694-704 (2018)

134. Diab, A. et al. Bempegaldesleukin (NKTR-214) plus nivolumab in patients with advanced solid tumors: phase I dose-escalation study of safety, efficacy, and immune activation (PIVOT-02). Cancer Discov. 10, 1158-1173 (2020).

135. Lotze, M. T. et al. High-dose recombinant interleukin 2 in the treatment of patients with disseminated cancer. Responses, treatment-related morbidity, and histologic findings. JAMA 256, 3117-3124 (1986).

136. Jackaman, C. et al. IL-2 intratumoral immunotherapy enhances $\mathrm{CD} 8^{+} \mathrm{T}$ cells that mediate destruction of tumor cells and tumor-associated vasculature: a nove mechanism for IL-2. J. Immunol. 171, 5051-5063 (2003).

137. Gutwald, J. G., Groth, W. \& Mahrle, G. Peritumoral injections of interleukin 2 induce tumour regression in metastatic malignant melanoma. Br. J. Dermatol. 130, 541-542 (1994).

138. Weide, B. et al. Survival after intratumoral interleukin-2 treatment of 72 melanoma patients and response upon the first chemotherapy during followup. Cancer Immunol. Immunother. 60, 487-493 (2011).

139. Charych, D. H. et al. NKTR-214, an engineered cytokine with biased IL2 receptor binding, increased tumor exposure, and marked efficacy in mouse tumor models. Clin. Cancer Res. 22, 680-690 (2016).

140. Bentebibel, S. E. et al. A first-in-human study and biomarker analysis of NKTR-214, a novel IL2R $\beta \gamma^{-}$ biased cytokine, in patients with advanced or metastatic solid tumors. Cancer Discov. 9, 711-721 (2019).

141. Mauldin, I. S. et al. Intratumoral interferon-gamma increases chemokine production but fails to increase $\mathrm{T}$ cell infiltration of human melanoma metastases. Cancer Immunol. Immunother. 65, 1189-1199 (2016)

142. Grob, J. J. et al. Large keratoacanthomas treated with intralesional interferon alfa-2a. J. Am. Acad. Dermatol. 29, 237-241 (1993).

143. Thestrup-Pedersen, K., Jacobsen, I. E. \& Frentz, G. Intralesional interferon-alpha $2 \mathrm{~b}$ treatment of basa cell carcinoma. Acta Derm. Venereol. 70, 512-514 (1990).
144. McDonald, R. R. \& Georgouras, K. Treatment of basal cell carcinoma with intralesional interferon alpha: a case report and literature review. Australas. J. Dermatol. 33, 81-86 (1992).

145. Hanlon, A., Kim, J. \& Leffell, D. J. Intralesional interferon alfa- $2 \mathrm{~b}$ for refractory, recurrent squamous cell carcinoma of the face. J. Am. Acad. Dermatol. 69 1070-1072 (2013).

146. Buechner, S. A. et al. Regression of basal cell carcinoma by intralesional interferon-alpha treatment is mediated by CD95 (Apo-1/Fas)-CD95 ligandinduced suicide. J. Clin. Invest. 100, 2691-2696 (1997).

147. Spaapen, R. M. et al. Therapeutic activity of high-dose intratumoral IFN-beta requires direct effect on the tumor vasculature. J. Immunol. 193, 4254-4260 (2014).

148. Santana Carrero, R. M. et al. IL-15 is a component of the inflammatory milieu in the tumor microenvironment promoting antitumor responses. Proc. Natl Acad. Sci. USA 116, 599-608 (2019).

149. Ugen, K. E et al. Regression of subcutaneous B16 melanoma tumors after intratumoral delivery of an IL-15-expressing plasmid followed by in vivo electroporation. Cancer Gene Ther. 13, 969-974 (2006).

150. Hu, Q. et al. Discovery of a novel IL-15 based protein with improved developability and efficacy for cancer immunotherapy. Sci. Rep. 8, 7675 (2018).

151. Bajetta, E. et al. Pilot study of subcutaneous recombinant human interleukin 12 in metastatic melanoma. Clin. Cancer Res. 4, 75-85 (1998)

152. Motzer, R. J. et al. Phase I trial of subcutaneous recombinant human interleukin-12 in patients with advanced renal cell carcinoma. Clin. Cancer Res. 4, 1183-1191 (1998).

153. Gollob, J. A. et al. Phase I trial of twice-weekly intravenous interleukin 12 in patients with metastatic renal cell cancer or malignant melanoma: ability to maintain IFN-gamma induction is associated with clinical response. Clin. Cancer Res. 6, 1678-1692 (2000).

154. Atkins, M. B. et al. Phase I evaluation of intravenous recombinant human interleukin 12 in patients with advanced malignancies. Clin. Cancer Res. 3, 409-417 (1997).

155. Algazi, A. et al. Intratumoral delivery of tavokinogene telseplasmid yields systemic immune responses in metastatic melanoma patients. Ann. Oncol. 31 532-540 (2020)

156. Algazi, A. P. et al. Phase II trial of IL-12 plasmid transfection and PD-1 blockade in immunologically quiescent melanoma. Clin. Cancer Res. 26 , 2827-2837 (2020)

157. Greaney, S. K. et al. Intratumoral plasmid IL12 electroporation therapy in patients with advanced melanoma induces systemic and intratumoral T-cell responses. Cancer Immunol. Res. 8, 246-254 (2020)

158. Bhatia, S. et al. Intratumoral delivery of plasmid IL12 via electroporation leads to regression of injected and noninjected tumors in Merkel cell carcinoma. Clin. Cancer Res. 26, 598-607 (2020).

159. Hewitt, S. L. et al. Intratumoral interleukin-12 mRNA therapy promotes TH1 transformation of the tumor microenvironment. Clin. Cancer Res. 26, 6284-6298 (2020).

160. Hamid, O. et al. Preliminary safety, antitumor activity and pharmacodynamics results of HIT-IT MEDI1191 (mRNA IL-12) in patients with advanced solid tumours and superficial lesions. Ann. Oncol. 32, S9-S13 (2021).

161. Li, Y. Z. et al. Multifunctional oncolytic nanoparticles deliver self-replicating IL-12 RNA to eliminate established tumors and prime systemic immunity. Nat. Cancer 1, 882- 893 (2020)

162. Kanca, H. et al. Intratumoral recombinant human interferon alpha-2a and vincristine combination therapy in canine transmissible venereal tumour. Vet. Med. Sci. 4, 364-372 (2018).

163. Vaquero, J. \& Martinez, R. Intratumoral immunotherapy with interferon-alpha and interleukin-2 in glioblastoma. Neuroreport 3 981-983 (1992)

164. Momin, N. et al. Anchoring of intratumorally administered cytokines to collagen safely potentiates systemic cancer immunotherapy. Sci. Transl Med. 11, eaaw2614 (2019).

165. Wagenaar, T. R. et al. Combinatorial treatment with intratumoral cytokine mRNAs results in high frequency of tumor rejection and development of anti-tumo immunity across a range of preclinical cancer models [abstract]. Cancer Res. 78 (Suppl. 13), LB130 (2018). 
166. Hewitt, S. L. et al. Durable anticancer immunity from intratumoral administration of IL-23, IL-36y, and OX40L mRNAs. Sci. Transl Med. 11, eaat9143 (2019).

167. Jimeno, A. et al. A phase $1 / 2$, open-label, multicenter, dose escalation and efficacy study of mRNA-2416, a lipid nanoparticle encapsulated mRNA encoding human OX40L, for intratumoral injection alone or in combination with durvalumab for patients with advanced malignancies [abstract]. Cancer Res. 80 (Suppl. 16), CT032 (2020).

168. Brahmer, J. R. et al. Phase I study of single-agent antiprogrammed death-1 (MDX-1106) in refractory solid tumors: safety, clinical activity, pharmacodynamics, and immunologic correlates. J. Clin. Oncol. 28, 3167-3175 (2010)

169. Herbst, R. S. et al. A study of MPDL3280A, an engineered PD-L1 antibody in patients with locally advanced or metastatic tumors [abstract]. J. Clin. Oncol. 31 (Suppl. 15), 3000 (2013).

170. Francis, D. M. et al. Blockade of immune checkpoints in lymph nodes through locoregional delivery augments cancer immunotherapy. Sci. Transl Med. 12 eaay3575 (2020)

171. Ascierto, P. A. et al. Ipilimumab $10 \mathrm{mg} / \mathrm{kg}$ versus ipilimumab $3 \mathrm{mg} / \mathrm{kg}$ in patients with unresectable or metastatic melanoma: a randomised, double-blind, multicentre, phase 3 trial. Lancet Oncol. 18, 611-622 (2017).

172. Sharma, P. et al. Nivolumab alone and with ipilimumab in previously treated metastatic urothelial carcinoma: Checkmate 032 Nivolumab $1 \mathrm{mg} / \mathrm{kg}$ Plus Ipilimumab $3 \mathrm{mg} / \mathrm{kg}$ expansion cohort results. J. Clin Oncol. 37, 1608-1616 (2019).

173. Marabelle, A., Kohrt, H. \& Levy, R. Intratumoral antiCTLA-4 therapy: enhancing efficacy while avoiding toxicity. Clin. Cancer Res. 19, 5261-5263 (2013).

174. van Hooren, L. et al. Local checkpoint inhibition of CTLA-4 as a monotherapy or in combination with antiPD1 prevents the growth of murine bladder cancer. Eur. J. Immunol. 47, 385-393 (2017).

175. Fransen, M. F., van der Sluis, T. C., Ossendorp, F. Arens, R. \& Melief, C. J. Controlled local delivery of CTLA-4 blocking antibody induces CD8 ${ }^{+}$T-celldependent tumor eradication and decreases risk of toxic side effects. Clin. Cancer Res. 19, 5381-5389 (2013)

176. Sandin, L. C. et al. Local CTLA4 blockade effectively restrains experimental pancreatic adenocarcinoma growth in vivo. Oncoimmunology 3, e27614 (2014)

177. Simmons, A. D. et al. Local secretion of anti-CTLA-4 enhances the therapeutic efficacy of a cancer immunotherapy with reduced evidence of systemic autoimmunity. Cancer Immunol. Immunother. 57, 1263-1270 (2008)

178. Marabelle, A. et al. Depleting tumor-specific Tregs at a single site eradicates disseminated tumors. J. Clin. Invest. 123, 2447-2463 (2013).

179. Ray, A. et al. A phase I study of intratumoral ipilimumab and interleukin-2 in patients with advanced melanoma. Oncotarget 7, 64390-64399 (2016)

180. Schwarze, J. K. et al. A phase I clinical trial on intratumoral and intracavitary administration of ipilimumab and nivolumab in patients with recurrent glioblastoma. J. Clin. Oncol. 38, 2534-2534 (2020).

181. Hamid, O. et al. First in human (FIH) study of an OX40 agonist monoclonal antibody (mAb) PF-04518600 (PF-8600) in adult patients (pts) with select advanced solid tumors: PRELIMINARY safety and pharmacokinetic (PK)/pharmacodynamic results [abstract]. J. Clin. Oncol. 34 (Suppl. 15), 3079 (2016).

182. Hansen, A. R. et al. A first-in-human phase I dose escalation study of the OX40 agonist MOXR0916 in patients with refractory solid tumors. Cancer Research 76 (Suppl. 14), CT097 (2016).

183. Bulliard, Y. et al. OX40 engagement depletes intratumoral Tregs via activating $\mathrm{F}_{\mathrm{C}} \gamma \mathrm{Rs}$, leading to antitumor efficacy. Immunol. Cell Biol. 92, 475-480 (2014)

184. Bulliard, Y. et al. Activating Fc gamma receptors contribute to the antitumor activities of immunoregulatory receptor-targeting antibodies. J. Exp. Med. 210, 1685-1693 (2013).

185. Suntharalingam, G. et al. Cytokine storm in a phase 1 trial of the anti-CD28 monoclonal antibody TGN 1412 N. Engl. J. Med. 355, 1018-1028 (2006).

186. Sagiv-Barfi, I. et al. Eradication of spontaneous malignancy by local immunotherapy. Sci. Trans/ Med. 10, eaan4488 (2018)

187. Vonderheide, R. H. et al. Clinical activity and immune modulation in cancer patients treated with
CP-870,893, a novel CD40 agonist monoclonal antibody. J. Clin. Oncol. 25, 876-883 (2007).

188. Kwong, B., Gai, S. A., Elkhader, J., Wittrup, K. D. \& Irvine, D. J. Localized immunotherapy via liposomeanchored anti-CD137+IL-2 prevents lethal toxicity and elicits local and systemic antitumor immunity. Cancer Res. 73, 1547-1558 (2013).

189. Dai, M., Yip, Y. Y., Hellstrom, I. \& Hellstrom, K. E. Curing mice with large tumors by locally delivering combinations of immunomodulatory antibodies. Clin. Cancer Res. 21, 1127-1138 (2015).

190. Sandin, L. C. et al. Locally delivered CD40 agonist antibody accumulates in secondary lymphoid organs and eradicates experimental disseminated bladder cancer. Cancer Immunol. Res. 2, 80-90 (2014).

191. Jackaman, C. \& Nelson, D. J. Intratumoral interleukin-2/agonist CD40 antibody drives CD4+independent resolution of treated-tumors and CD4 dependent systemic and memory responses. Cancer Immunol. Immunother. 61, 549-560 (2012)

192. Van De Voort, T. J., Felder, M. A., Yang, R. K., Sondel, P. M. \& Rakhmilevich, A. L. Intratumoral delivery of low doses of anti-CD 40 mAb combined with monophosphoryl lipid a induces local and systemic antitumor effects in immunocompetent and T celldeficient mice. J. Immunother 36, 29-40 (2013).

193. van Mierlo, G J et al. CD40 stimulation leads to effective therapy of $\mathrm{CD}^{4} 0^{-}$tumors through induction of strong systemic cytotoxic T lymphocyte immunity. Proc. Natl Acad. Sci. USA 99, 5561-5566 (2002).

194. Tselikas, L. et al. Pickering emulsions with ethiodized oil and nanoparticles for slow release of intratumoral anti-CTLA4 immune checkpoint antibodies. J. Immunother. Cancer 8, e000579 (2020).

195. Ishihara, J. et al. Matrix-binding checkpoint immunotherapies enhance antitumor efficacy and reduce adverse events. Sci. Transl Med. 9, eaan0401 (2017).

196. Bol, K. F., Schreibelt, G., Gerritsen, W. R., de Vries, I. J. $\&$ Figdor, C. G. Dendritic cell-based immunotherapy: state of the art and beyond. Clin. Cancer Res. 22 1897-1906 (2016).

197. Melero, I., Vile, R. G. \& Colombo, M. P. Feeding dendritic cells with tumor antigens: self-service buffet or a la carte? Gene Ther. 7, 1167-1170 (2000).

198. Mazzolini, G. et al. Intratumoral injection of dendritic cells engineered to secrete interleukin- 12 by recombinant adenovirus in patients with metastatic gastrointestinal carcinomas. J. Clin. Oncol. 23, 999-1010 (2005).

199. Huarte, E. et al. Intratumoural administration of dendritic cells: hostile environment and help by gene therapy. Expert Opin. Biol. Ther. 5, 7-22 (2005).

200. Karlsson-Parra, A. et al. Ilixadencel - an allogeneic cell-based anticancer immune primer for intratumoral administration. Pharm. Res. 35, 156 (2018).

201. Rizell, M. et al. Phase 1 trial with the cell-based immune primer ilixadencel, alone, and combined with sorafenib, in advanced hepatocellular carcinoma. Front. Oncol. 9, 19 (2019)

202. Frobom, R. et al. Phase I trial evaluating safety and efficacy of intratumorally administered inflammatory allogeneic dendritic cells (ilixadencel) in advanced gastrointestinal stromal tumors. Cancer Immunol. Immunother. 69, 2393-2401 (2020).

203. Etxeberria, I. et al. Intratumor adoptive transfer of IL-1 2 mRNA transiently engineered antitumor CD8 T cells. Cancer Cell 36, 613-629.e7 (2019).

204. Minute, L. et al. Cellular cytotoxicity is a form of immunogenic cell death. J. Immunother. Cancer 8 e000325 (2020)

205. Dillman, R. O. et al. Intralesional lymphokine-activated killer cells as adjuvant therapy for primary glioblastoma. J. Immunother. 32, 914-919 (2009).

206. June, C. H., O'Connor, R. S., Kawalekar, O. U., Ghassemi, S. \& Milone, M. C. CAR T cell immunotherapy for human cancer. Science 359 . 1361-1365 (2018).

207. Brown, C. E. et al. Regression of glioblastoma after chimeric antigen receptor T-cell therapy. N. Engl. J. Med. 375, 2561-2569 (2016)

208. Brown, C. E. et al. Bioactivity and safety of IL13Ra2redirected chimeric antigen receptor $\mathrm{CD}^{+} \mathrm{T}$ cells in patients with recurrent glioblastoma. Clin. Cancer Res. 21, 4062-4072 (2015)

209. Etxeberria, I. et al. Engineering bionic T cells: signal 1 , signal 2 , signal 3 , reprogramming and the removal of inhibitory mechanisms. Cell Mol. Immunol. 17 , 576-586 (2020)

210. Adusumilli, P. S. et al. Regional delivery of mesothelintargeted CAR T cells for pleural cancers: safety and preliminary efficacy in combination with anti-PD-1 agent [abstract]. J. Clin. Oncol. 37 (Suppl. 15), 2511 (2019).

211. Papa, S. et al. A phase I trial of T4 CAR T-cell immunotherapy in head and neck squamous cancer (HNSCC) [abstract]. J. Clin. Oncol. 36 (Suppl. 15), 3046 (2018).

212. Katz, S. C. et al. HITM-SURE: Hepatic immunotherapy for metastases phase Ib anti-CEA CAR-T study utilizing pressure enabled drug delivery. J. Immunother. Cancer 8, e001097 (2020)

213. Goebeler, M. E. \& Bargou, R. C. T cell-engaging therapies - BiTEs and beyond. Nat. Rev. Clin. Oncol. 17, 418-434 (2020)

214. Topp, M. S. et al. Targeted therapy with the T-cellengaging antibody blinatumomab of chemotherapyrefractory minimal residual disease in B-lineage acute lymphoblastic leukemia patients results in high response rate and prolonged leukemia-free survival. J. Clin. Oncol. 29, 2493-2498 (2011).

215. Linke, R., Klein, A. \& Seimetz, D. Catumaxomab: clinical development and future directions. $m A b s \mathbf{2}$, 129-136 (2010)

216. Heiss, M. M. et al. The trifunctional antibody catumaxomab for the treatment of malignant ascites due to epithelial cancer: results of a prospective randomized phase II/III trial. Int. J. Cancer 127, 2209-2221 (2010).

217. Burges, A. et al. Effective relief of malignant ascites in patients with advanced ovarian cancer by a trifunctional anti-EpCAM $\times$ anti-CD3 antibody: a phase I/II study. Clin. Cancer Res. 13, 3899-3905 (2007)

218. Bacac, M., Klein, C. \& Umana, P. CEA TCB: a novel head-to-tail 2:1 $\mathrm{T}$ cell bispecific antibody for treatment of CEA-positive solid tumors. Oncoimmunology 5 , e1203498 (2016)

219. Damato, B. E., Dukes, J., Goodall, H. \& Carvajal, R. D. Tebentafusp: $T$ cell redirection for the treatment of metastatic uveal melanoma. Cancers 11, 971 (2019).

220. Wong, B., Arron, J. \& Choi, Y. T cell receptor signals enhance susceptibility to Fas-mediated apoptosis. J. Exp. Med. 186, 1939-1944 (1997)

221. Claus, C et al. Tumor-targeted 4-1BB agonists for combination with $\mathrm{T}$ cell bispecific antibodies as off-theshelf therapy. Sci. Transl Med. 11, eaav5989 (2019).

222. Lakins, M. A. et al. FS222, a CD137/PD-L1 tetravalent bispecific antibody, exhibits low toxicity and antitumor activity in colorectal cancer models. Clin. Cancer Res. 26, 4154-4167 (2020).

223. Voeller, J. et al. Combined innate and adaptive immunotherapy overcomes resistance of immunologically cold syngeneic murine neuroblastoma to checkpoint inhibition. J. Immunother. Cancer $\mathbf{7}$, 344 (2019).

224. Navid, F. et al. Phase I trial of a novel anti-GD2 monoclonal antibody, Hu14.18K322A, designed to decrease toxicity in children with refractory or recurrent neuroblastoma. J. Clin. Oncol. 32 1445-1452 (2014).

225. Federico, S. M. et al. A pilot trial of humanized antiGD2 monoclonal antibody (hu14.18K322A) with chemotherapy and natural killer cells in children with recurrent/refractory neuroblastoma. Clin. Cancer Res. 23, 6441-6449 (2017).

226. Shusterman, S. et al. Antitumor activity of hu14.18-IL2 in patients with relapsed/refractory neuroblastoma: a Children's Oncology Group (COG) phase II study. J. Clin. Oncol. 28, 4969-4975 (2010).

227. Yu, A. L. et al. Anti-GD2 antibody with GM-CSF, interleukin-2, and isotretinoin for neuroblastoma N. Engl. J. Med. 363, 1324-1334 (2010)

228. Johannsen, M. et al. The tumour-targeting human L19-IL2 immunocytokine: preclinical safety studies, phase I clinical trial in patients with solid tumours and expansion into patients with advanced renal cell carcinoma. Eur. J. Cancer 46, 2926-2935 (2010).

229. Danielli, R et al. Intralesional administration of L19-IL2/L19-TNF in stage III or stage IVM1 melanoma patients: results of a phase II study. Cancer Immunol. Immunother. 64, 999-1009 (2015).

230. Heaton, K. M., Ju, G. \& Grimm, E. A. Human interleukin 2 analogues that preferentially bind the intermediate-affinity interleukin 2 receptor lead to reduced secondary cytokine secretion: implications for the use of these interleukin 2 analogues in cancer immunotherapy. Cancer Res. 53, 2597-2602 (1993)

231. Klein, C. et al. Cergutuzumab amunaleukin (CEA-IL2v), a CEA-targeted IL-2 variant-based immunocytokine for combination cancer immunotherapy: overcoming limitations of aldesleukin and conventional IL-2-based immunocytokines. Oncoimmunology 6, e1277306 (2017). 
232. Soerensen, M. M. et al. Safety, PK/PD, and anti-tumor activity of RO6874281, an engineered variant of interleukin-2 (IL-2v) targeted to tumor-associated fibroblasts via binding to fibroblast activation protein (FAP). J. Clin. Oncol. 36, e15155 (2018).

233. Fedele, V. \& Melisi, D. Permissive state of EMT: the role of immune cell compartment. Front. Oncol. 10, 587 (2020).

234. Strauss, J. et al. Phase I evaluation of M7824, a bifunctional fusion protein targeting TGF- $\beta$ and PD-L1, in patients with human papillomavirus (HPV)-associated malignancies [abstract]. Cancer Res. 79 (Suppl. 13), CT075 (2019).

235. Fallon, J. et al. The immunocytokine NHS-IL12 as a potential cancer therapeutic. Oncotarget 5 1869-1884 (2014).

236. Teijeira, A. et al. CXCR1 and CXCR2 chemokine receptor agonists produced by tumors induce neutrophil extracellular traps that interfere with immune cytotoxicity. Immunity 52, 856-871.e8 (2020).

237. Strauss, J. et al. First-in-human phase I trial of a tumor-targeted cytokine (NHS-IL12) in subjects with metastatic solid tumors. Clin. Cancer Res. 25, 99-109 (2019).

238. Autio, K. A., Boni, V., Humphrey, R. W. \& Naing, A. Probody therapeutics: an emerging class of therapies designed to enhance on-target effects with reduced off-tumor toxicity for use in immuno-oncology. Clin. Cancer Res. 26, 984-989 (2020).

239. Kamata-Sakurai, M. et al. Antibody to CD137 activated by extracellular adenosine triphosphate is tumor selective and broadly effective in vivo without systemic immune activation. Cancer Discov. 11 158-175 (2021)

240. Aznar, M. A. et al. Intratumoral delivery of immunotherapy-act locally, think globally. J. Immunol. 198, 31-39 (2017)

241. Hong, W. X. et al. Intratumoral immunotherapy for early-stage solid tumors. Clin. Cancer Res. 26 3091-3099 (2020)

242. Melero, I. P. et al. Repurposing infectious disease vaccines for intratumoral immunotherapy. J. Immunother. Cancer 8, e000443 (2020)

243. Newman, J. H. et al. Intratumoral injection of the seasonal flu shot converts immunologically cold tumors to hot and serves as an immunotherapy for cancer Proc. Natl Acad. Sci. USA 117, 1119-1128 (2020).

244. Shekarian, T. et al. Repurposing rotavirus vaccines for intratumoral immunotherapy can overcome resistance to immune checkpoint blockade. Sci. Trans/ Med. 11 eaat5025 (2019)

245. Aznar, M. A. et al. Repurposing the yellow fever vaccine for intratumoral immunotherapy. EMBO Mol. Med. 12, e10375 (2020)

246. Galluzzi, L., Buque, A., Kepp, O., Zitvogel, L. \& Kroemer, G. Immunogenic cell death in cancer and infectious disease. Nat. Rev. Immunol. 17, 97-111 (2017)

247. Kroemer, G., Galluzzi, L., Kepp, O. \& Zitvogel, L. Immunogenic cell death in cancer therapy. Annu. Rev. Immunol. 31, 51-72 (2013).

248. Pol, J. et al. Trial Watch: immunogenic cell death inducers for anticancer chemotherapy. Oncoimmunology 4, e1008866 (2015).

249. Burtness, B. et al. Pembrolizumab alone or with chemotherapy versus cetuximab with chemotherapy for recurrent or metastatic squamous cell carcinoma of the head and neck (KEYNOTE-048): a randomised, open-label, phase 3 study. Lancet 394, 1915-1928 (2019).

250. Gandhi, L. et al. Pembrolizumab plus chemotherapy in metastatic non-small-cell lung cancer. N. Engl. J. Med. 378, 2078-2092 (2018)

251. Halmos, B et al. A matching-adjusted indirect comparison of pembrolizumab+chemotherapy vs. nivolumab+ipilimumab as first-line therapies in patients with PD-L1 TPS $\geq 1 \%$ metastatic NSCLC. Cancers 12, 3648 (2020).

252. He, X. et al. Upfront dose-reduced chemotherapy synergizes with immunotherapy to optimize chemoimmunotherapy in squamous cell lung carcinoma. J. Immunother. Cancer 8, e000807 (2020).

253. Swami, U. et al. Exceptional responses with sequential metronomic temozolomide after pembrolizumab failure in patients with metastatic melanoma. Melanoma Res. 29, 643-647 (2019).

254. Pfirschke, C. et al. Immunogenic chemotherapy sensitizes tumors to checkpoint blockade therapy. Immunity 44, 343-354 (2016).

255. Casares, N. et al. Caspase-dependent immunogenicity of doxorubicin-induced tumor cell death. J. Exp. Med. 202, 1691-1701 (2005)

256. Oratz, R et al. Intratumoral cisplatin/adrenaline injectable gel for the treatment of patients with cutaneous and soft tissue metastases of malignant melanoma. Melanoma Res. 13, 59-66 (2003)

257. Vogl, T. J. et al. CT-guided intratumoural administration of cisplatin/epinephrine gel for treatment of malignant liver tumours. Br. J. Cancer 86, 524-529 (2002)

258. Khan, F., Anker, C. J., Garrison, G. \& Kinsey, C. M Endobronchial ultrasound-guided transbronchial needle injection for local control of recurrent non-small cell lung cancer. Ann. Am. Thorac. Soc. 12, 101-104 (2015).

259. Mori, V., Roy, G. S., Bates, J. H. T. \& Kinsey, C. M Cisplatin pharmacodynamics following endobronchial ultrasound-guided transbronchial needle injection into lung tumors. Sci. Rep. 9, 6819 (2019).

260. Hohenforst-Schmidt, W. et al. Intratumoral chemotherapy for lung cancer: re-challenge current targeted therapies. Drug Des. Devel Ther. 7, 571-583 (2013).

261. Marabelle, A. et al. Starting the fight in the tumor: expert recommendations for the development of human intratumoral immunotherapy (HIT-IT) Ann. Oncol. 29, 2163-2174 (2018).

262. Goldmacher, G. V. et al. Response criteria for intratumoral immunotherapy in solid tumors: itRECIST. J. Clin. Oncol. 38, 2667-2676 (2020).

263. Champiat, S. et al. Intratumoral immunotherapy: from trial design to clinical practice. Clin. Cancer Res. 27, 665-679 (2021).

\section{Acknowledgements}

The authors thank the patients, the patients' families and colleagues who have participated in the authors' preclinical and clinical research projects on intratumoural immunotherapy and have contributed to the development of the authors expertise in that field. The authors thank in particular T. de Baere, L. Tselikas, S. Ammari, S. Farhane and C. Massard for their contribution to the implementation of intratumoural immunotherapy trials at Gustave Roussy. The authors also thank their colleagues at the University of Navarra, especially
A. Benito, M. Rodriguez-Ruiz, M.F. Sanmamed, C. de Andrea, J.L. Perez-Gracia, M. Ponz, L. Resano, I. Coicoechea and M. Egaña. The work of M.A. is funded by the Asociación Española contra el Cancer (AECC) Foundation, and the authors acknowledge continued financial support from the Spanish Ministry of Economy and Competitiveness (MINECO SAF2014-52361-R and SAF 2017-83267-C2-1R (AEI/FEDER,UE)), the Cancer Research Institute under the CRI-CLIP, the AECC Foundation under grant GCB 15152947MELE, the Joint Translational Call for Proposals 2015 (JTC 2015) TRANSCAN-2 (code TRS715 2016-00000371), and the European Commission within the Horizon 2020 Programme (PROCROP - 718 635122).

\section{Author contributions}

The authors contributed equally to all aspects of the article.

\section{Competing interests}

I.M. has received research grants from Alligator, Bioncotech, Bristol Myers Squibb (BMS), Leadartis, Pfizer and Roche; has received speaker's bureau honoraria from MSD; and is a consultant or advisory board member for Alligator AstraZeneca, Bayer, Bioncotech, BMS, F-Star, Genmab, Gossamer, Merck Serono, Numab, Pieris and Roche. E.C. is a consultant or advisory board member for AstraZeneca, Beigene, BMS, MSD and Roche. S.C. has received honoraria from Amgen, AstraZeneca, BMS, Janssen, Merck, MSD, Novartis and Roche; is an advisory board member for Amgen and AstraZeneca; has received funding for travel and conference attendance from AstraZeneca, MSD and Roche; and has received research grants from AstraZeneca, BMS, Boehringer Ingelheim, Janssen-Cilag, Merck, Novartis, Onxeo, Pfizer, Roche and Sanofi, and non-financial research support (investigational drugs) from AstraZeneca, Bayer, BMS, Boehringer Ingelheim, Medimmune, Merck, NH TherAGuiX, Onxeo, Pfizer and Roche. S.C. has been a principal investigator of academic or industry-sponsored clinical trials of intratumoural immunotherapies for Abbvie, AstraZeneca/ Medimmune, BMS, Eisai/H3 Biomedicine, IDERA, Lytix Biopharma, MSD, Nanobiotix and Sanofi/BioNTech. A.M. has been a principal investigator of academic or industry-sponsored clinical trials of intratumoural immunotherapies from AstraZeneca, BMS, Eisai, IDERA, Lytix Biopharma, Merck/MSD, Roche and Transgene; is a member of the Data Safety and Monitoring Board of a trial of a intratumoural TLR3 agonist sponsored by Oncovir (NCT02423863); and has participated in scientific advisory boards or has provided consultancy services on the topic of intratumoural immunotherapies for Amgen, AstraZeneca, Banque Pour l'Investissement, Bayer, Eisai, eTheRNA, Lytix Biopharma, Medincell, MSD, Novartis, Oncosec, Pillar Partners, Rigontec and Sanofi/BioNTech. M.A. declares no competing interests.

\section{Peer review information}

Nature Reviews Clinical Oncology thanks H. Kaufman and the other, anonymous, reviewer(s) for their contribution to the peer review of this work.

\section{Publisher's note}

Springer Nature remains neutral with regard to jurisdictional claims in published maps and institutional affiliations.

\section{Supplementary information}

The online version contains supplementary material available at https://doi.org/10.1038/s41571-021-00507-y.

(c) Springer Nature Limited 2021 